Published in final edited form as:

Nat Neurosci. 2019 October ; 22(10): 1635-1648. doi:10.1038/s41593-019-0486-0.

\title{
Fragmented mitochondria released from microglia trigger A1 astrocytic response and propagate inflammatory neurodegeneration
}

\author{
Amit U. Joshi ${ }^{1}$, Paras S. Minhas ${ }^{2}$, Shane A. Liddelow ${ }^{3}$, Bereketeab Haileselassie ${ }^{1,4}$, Katrin \\ I. Andreasson ${ }^{2}$, Gerald W. Dorn II ${ }^{5}$, Daria Mochly-Rosen ${ }^{1, *}$ \\ ${ }^{1}$ Department of Chemical and Systems Biology, Stanford University School of Medicine, Stanford, \\ CA; \\ ${ }^{2}$ Department of Neurology \& Neurological Sciences, Stanford School of Medicine, Stanford, CA; \\ ${ }^{3}$ Department of Neurobiology, Stanford University, School of Medicine, Stanford, CA (Current \\ address: Department of Neuroscience and Physiology; Neuroscience Institute; Department of \\ Neuroscience and Physiology, NYU Langone Medical Center, NY; Department of Pharmacology \\ and Therapeutics, The University of Melbourne, Melbourne, Australia); \\ ${ }^{4}$ Department of Pediatrics division of Critical Care Medicine, Stanford University School of \\ Medicine, Stanford, CA; \\ ${ }^{5}$ Center for Pharmacogenomics, Department of Internal Medicine, Washington University School \\ of Medicine, St. Louis MO USA
}

\begin{abstract}
In neurodegenerative diseases, debris of dead neurons are thought to trigger glia-mediated neuroinflammation, thus increasing neuronal death. Here, we show that expression of neurotoxic proteins associated with these diseases in microglia alone is sufficient to trigger death of naïve neurons directly and to propagate neuronal death through activation of naïve astrocytes to A1 state. Injury propagation is mediated, in great part, by the release of fragmented and dysfunctional microglial mitochondria to the neuronal milieu. The amount of damaged mitochondria released from microglia relative to functional mitochondria and the consequent neuronal injury are determined by Fis1-mediated mitochondrial fragmentation within the glia cells. The propagation of inflammatory response and neuronal cell death by extracellular dysfunctional mitochondria
\end{abstract}

\footnotetext{
Users may view, print, copy, and download text and data-mine the content in such documents, for the purposes of academic research, subject always to the full Conditions of use:http://www.nature.com/authors/editorial_policies/license.html\#terms

*Corresponding author: Daria Mochly-Rosen, Department of Chemical \& Systems Biology, School of Medicine, Stanford University, CA, USA, mochly@stanford.edu Tel: 650-255-1053.

Author Contributions

A.U.J and D.M-R generated the hypothesis and experimental design. A.U.J, D.M-R and G.W.D contributed to manuscript preparation. K.I.A contributed to experimental design. A.U.J performed isolations of neurons, microglia and astrocytes, collected, analyzed and interpreted the results from earlier mouse studies, qPCR, ELISA, immunoblots, and mitochondrial assays. P.S.M. performed isolations of human MDMs, neurons, microglia and astrocytes, collected data from seahorse experiments and helped with data analysis. B.H. performed mitochondrial assays, ELISA and immunoblots and helped with data analysis. S.A.L isolated microglia and performed Microfluidic qPCR and helped with data analysis. All the authors reviewed and edited the manuscript.

Competing Interests

Patents on P110 and its utility in HD, ALS and other neurodegenerative diseases have been filed by D.M-R and A.U.J. The other authors declare that they have no conflict of interest.
} 
suggests a potential new intervention for neurodegeneration - one that inhibits mitochondrial fragmentation in microglia, thus inhibiting the release of dysfunctional mitochondria into the extracellular milieu of the brain, without affecting the release of healthy neuroprotective mitochondria.

\section{Keywords}

Mitochondrial dynamics; neural inflammation; Fis1; P110; dynamin related protein 1; neurodegenerative disease

A common feature of neurodegenerative diseases, such as Alzheimer's disease (AD), amyotrophic lateral sclerosis (ALS) and Huntington's disease (HD), is accumulation of neurotoxic proteins in the neurons that leads to neuronal dysfunction and eventually to neuronal death ${ }^{1}$. Although, other cells, including microglia and astrocytes (glial cells) in the brain, also express these proteins and therefore may be damaged directly, the contribution of this potential glial damage to neuronal injury is not fully understood. Much research indicates that the role of glia cells in the brain is neuroprotective in nature; these cells release trophic factors, protect against oxidative stress and excitotoxity, provide essential metabolites and remove damaging agents and cell debris in the brain's milieu ${ }^{2-4}$. However, in neurodegenerative diseases, glia are activated to a state that triggers increased secretion of proinflammatory factors, leading to further neurotoxicity through a mechanism collectively termed neuroinflammation ${ }^{5-8}$. This glial activation and the consequent neuronal damage is attributed to unchecked glial stimulation by exposure to neuronal debris ${ }^{8}$. In animal models, the activated state of astrocytes, termed A1 state, is rapidly induced following brain injury by activated microglia, and causes further neuronal injury and cell death ${ }^{9}$. However, what is the trigger for this unchecked neuroinflammatory response and whether there are pharmacological agents to prevent A1 astrocyte formation and block neuronal injury have yet to be determined ${ }^{9}$. Here we set out to address these questions.

There is a clear role for excessive mitochondrial fragmentation and dysfunction in neurons in neurodegenerative diseases, including in HD, AD, ALS, Parkinson's disease, dementia, ataxia, and hypertension-induced encephalopathy ${ }^{10-13}$. Indeed, mitochondrial fragmentation as a consequence of excessive dynamin-related protein 1 (Drp1)-induced mitochondrial fission is a prototypical feature of these experimental and clinical neurodegenerative diseases ${ }^{14-16}$. Interrupting excessive Drp1-mediated mitochondrial fission can prevent neuronal degeneration ${ }^{17}$. A heptapeptide (P110) that selectively inhibits the binding of activated Drp1 to one of its mitochondrial receptors, Fis1, without affecting physiological mitochondrial fission ${ }^{16}$ is neuroprotective in patients-derived cells and in genetic mouse models of AD, HD and ALS ${ }^{12,18-20}$. We therefore determined whether Drp1/Fis1-mediated excessive mitochondrial fission contributes to glial activation in mouse models of neurodegenerative diseases. 


\section{Results}

\section{Glia activation in models of neurodegenerative diseases is inhibited by P110, a selective inhibitor of excessive mitochondrial fission and fragmentation}

Using samples from the same mice described in our previously published studies ${ }^{12,18-20}$, we found evidence for activation of both microglia and astrocytes in murine models of $\mathrm{AD}, \mathrm{HD}$ and ALS (Fig. 1) when measured at the end of the study for each cohort (end point in Fig. 1a). We reported that sustained treatment with P110 (the timing and duration of which are indicated by the maroon bars in Fig. 1a) slowed down disease progression in 5XFAD mice (AD model; expressing human APP and PSEN1 transgenes with a total of five AD-linked mutations: the Swedish (K670N/M671L), Florida (I716V), and London (V717I) mutations in APP, and the M146L and L286V mutations in PSEN1) significantly decreased in A $\beta$ levels, improved mitochondrial health and short term/long term recall in an open field test ${ }^{18}$. Sustained P110 treatment also significantly increased life span of in SOD1G93A mice (ALS model; survival after paralysis: Vehicle $=21$ days; $P 110=41.5$ days; $p$-value:0.0039) ${ }^{12}$. Finally, sustained P110 treatment also improved the life span of R6/2 mice (HD model; dead/survival at 13 weeks; Vehicle $=6 / 12 ; P 110=1 / 12 ; p$-value:0.0006) ${ }^{19,20}$. Here we show that this sustained P110 treatment also inhibited neuroinflammation in these models, as indicated by decreased levels of activated astrocytes and microglia in histological studies, measured by brain levels of GFAP and Iba-1, respectively (Fig. 1b-d and Supplementary Fig. 1). Expression of proinflammatory genes (Fig. 1e) and inflammatory cytokines accumulation in the tissue (Fig. 1f $-\mathrm{j}$ ) also decreased relative to vehicle-treated mice in each of neurodegenerative disease mouse models but remained higher than levels of age-matched wildtype (WT) control mice. These data indicate a role for Drp1/Fis1-mediated excessive mitochondrial fission in neuroinflammation associated with these neurodegenerative diseases. [Note that at the time of samples' collection (end point in Fig. 1a) in each model of neurodegenerative diseases, $10 \%$ of the P110-treated mice died, none of the matched WT or the 5XFAD mice died in any of the treatment groups, but $\sim 40 \%$ of R6/2 and ALS controltreated mice died ${ }^{12,18-20}$.]

\section{Microglia are directly activated by neurotoxic proteins in a mechanism dependent on Drp1/ Fis1-mediated excessive mitochondrial fission}

P110 treatment in vivo may delay neuronal loss which, in turn, reduces neuronal debrisinduced gliosis or P110 treatment may directly inhibit glial activation. To examine these two possible mechanisms for P110's anti-inflammatory effect, we moved to in vitro studies in which microglia were exposed to neurotoxic proteins in the absence of neurons. Expression of a long track of neurotoxic poly glutamine (Q73) as a model for HD in the microglial cell line, BV2, was sufficient to trigger mitochondrial fragmentation and dysfunction (Fig. 2a,b), and provoked P110-inhibitable neuroinflammatory responses in microglia, as measured by inflammasome formation, increased cellular reactive oxygen species (ROS) and decline in mitochondrial function (Fig. 2c-e), as compared with cells expressing the non-pathological poly glutamine, Q23. The levels of the proinflammatory cytokines, tissue necrosis factor a (TNFa) and interleukin $1 \beta$ (IL-1 $\beta$ ), increased $\sim 10$ folds by expressing Q73 in these microglia and P110 treatment blunted the increases by more than $50 \%$ (Fig. 2f). We confirmed that P110 treatment of microglia expressing Q73 inhibited Drp1 activation, as 
measured by inhibition of Drp1 accumulation in the mitochondrial fraction and decreasing the ratio of Drp1 phosphorylation on the activating site (S616) to that on the inactivating site $(\mathrm{S} 637)^{21}$ (Fig. 2g, h). Indicating that the effects above were not due simply to the transient transfection BV2, the responses of BV2 microglia to expression of Q73 were similar to those observed in primary microglia isolated from R6/2 mice, a murine model of $\mathrm{HD}^{22}$ (Fig. 2i-k). As expected, mitochondrial dysfunction and cytokine release were lower when using microglia of WT littermate relative microglia of R6/2 mice (Fig. 2i-k). To confirm that these primary microglia are functional, we also stimulated them with the bacterial-derived lipopolysaccharide (LPS) and found a further decline in ATP production, greater levels of cellular and mitochondrial reactive oxygen species (ROS) and a much greater cytokine release in R6/2-derived microglia relative to microglia derived from WT littermates (Fig. 2ik); all markers of microglial activation were significantly reduced when excessive mitochondrial fission was inhibited by P110. Similar effects of blunting activation by inhibiting Drp1/Fis1 interactions were observed when using primary rat microglia stimulated with $0.1 \mu \mathrm{g} / \mathrm{ml}$ of LPS alone (Supplementary Fig. 2) or with $0.01 \mu \mathrm{g} / \mathrm{ml}$ of LPS together with nigericin (an induced of NACHT, LRR and PYD domains-containing protein 3, NLRP3, inflammasome ${ }^{23}$; Fig. 21-o), used in a two-signal model of inflammation in culture.

The signature of microglia activation was not unique to models of HD. Expression of the mutant SOD1 G93A in BV2 microglia, as a model of $\mathrm{ALS}^{24}$, or treating microglia with oligomeric amyloid $\beta$ peptide- $42\left(\mathrm{oA} \beta_{42}\right)$, as a model for $\mathrm{AD}^{25}$, also triggered mitochondrial fragmentation, and dysfunction, decreasing ATP levels production, increasing mitochondrial and cell reactive oxygen species (ROS) production, and decreasing oxidative phosphorylation in the microglia (Fig. 3a-d and $\mathrm{f}-\mathrm{i}$, respectively). These treatments also increased cytokine production and inflammasome formation on mitochondria of the activated BV2 microglia (Fig. 3e and j, respectively) and all these effects were significantly blunted by inhibiting Fis1/Drp1 interaction through treatment of the microglia with P110 (Fig. 3e, j, k). Together, these data show that microglial activation with neurotoxic proteins is indifferent from that by the classical activator of inflammatory response, LPS, and that this microglial activation is dependent on Drp1/Fis1-induced excessive mitochondrial fission.

\section{Microglia propagate pro-inflammatory response to activate astrocytes}

Astrocytes, the other subset of glial cells in the brain, have a wide range of important physiological roles, including neuroprotective roles, when in A2 state ${ }^{9}$, and neuroinflammatory roles, when activated to A1 state ${ }^{9}$. Because we noted activation of astrocytes in the murine models of AD, HD and ALS that correlated with severity of the disease phenotypes (Fig. 1, Supplementary Fig. 1 and published work ${ }^{12,18,19}$ ), we assessed possible microglial-astrocyte cross-talk. We activated cultured microglia in the presence or absence of P110, as in figures 2 and 3, by expressing Q73 or mutant G93A SOD1 (model of HD ALS, respectively; Fig. 4a, g; schemes), or used primary microglia from R6/2 mice and WT littermate (Fig. 4n, scheme). After culturing for $24 \mathrm{hrs,} \mathrm{we} \mathrm{transferred} \mathrm{the} \mathrm{microglial-}$ conditioned media (MCM) to naïve primary astrocytes (Fig. 4a-n) or to R6/2-derived astrocytes and their littermate WT astrocytes (Fig. 4o). Transfer of BV2 microglial- 
conditioned media of Q73 (HD model) or SOD1 A93G (ALS model) to primary astrocytes induced pathological mitochondrial fragmentation in the astrocytes, evoked mitochondrial dysfunction, as evidenced by lower ATP levels, loss of normal inner mitochondrial membrane polarization, increase in mitochondrial ROS production and increased cell death (Fig. 4a-e, and. Fig. 4g-k for HD and ALS, respectively). These treatments resulted in astrocyte activation to the A1, proinflammatory state (Fig. 4 f and. Fig. 41 for HD and ALS, respectively) and many fold increased release of the proinflammatory cytokines, TNFa and IL-1 $\beta$ (Fig. 4m). All these effects were blunted when the microglia alone were treated with P110, 24 hrs prior to transferring their media to the naïve astrocytes. As the half-life of P110 is about 15 minutes in culture, the data suggest that Drp1/Fis1 fragmentation in microglia generated a diffusible signal to the astrocytes, resulting in astrocytes' activation to the A1 state.

Importantly, indicating that propagation of the inflammatory response is not simply due to the genetic manipulation of the BV2 microglial cell line, a two-fold stronger propagation of the pro-inflammatory signal to astrocytes was also induced by transferring conditioned media from R6/2 primary microglia relative to primary microglia from WT mice; there was an increased mitochondrial and cell ROS levels in naive astrocytes and even higher cellular ROS in primary astrocytes isolated from R6/2 mice (Fig. 4n). The propagation of the R6/2 microglia signal to astrocytes was exacerbated by LPS treatment of the donor microglia and was blunted by treatment of the donor microglia with P110 (Fig. 4n). Increased cellular and mitochondrial ROS were found also when the proinflammatory microglial media were transferred to R6/2-derived astrocytes (Fig. 4o) and the rise in cellular ROS was many folds higher relative to media transfer to astrocytes of WT littermates (Fig. 4o vs. n). Here too, we confirmed that the activation of the mouse astrocytes was due to canonical activation of microglia; treatment of the microglia with bacterial LPS and transfer of the conditioned media to astrocytes (Fig. 4n, o and Supplementary Fig. 3). Primary rat microglia similarly propagated inflammatory response in primary rat astrocytes by LPS/nigericin (Fig. 5a-f) or by direct treatment of the astrocytes with the pro-inflammatory cytokines TNFa, IL-1 $a$ and $\mathrm{C} 1 \mathrm{q}^{9}$ (Fig. 5g-1), which generated similar activation responses in astrocytes thus serving as positive controls for general immune activation of the astrocytes ${ }^{9}$. Therefore, propagation of the activation signal from all types of microglia to naive astrocytes or to R6/2 primary astrocytes was dependent upon Drp1/Fis1-mediated mitochondrial fission in the microglia; it was inhibited when the microglia were treated with P110 24 hrs prior to transfer of their conditioned media to the astrocytes (Fig. 4n,o, Fig. 5 and Supplementary Fig. 3).

Treatment of astrocytes with P110 directly also inhibited their transition to the proinflammatory A1 state by direct stimulation with TNFa, IL-1a and C1q (Fig. 5g-1), indicating that Drp1/Fis1-mediated mitochondrial fragmentation also plays a critical role in activation of astrocytes. Treatment of the astrocytes with a small molecule inhibitor of Drp1, Mdivi-1, or a peptide inhibitor of Drp1/Mff-mediated physiological fission, P259 ${ }^{26}$, exerted similar effects on TNFa, IL-1a and C1q-induced mitochondrial fragmentation (Fig. 5g; quantitated on the right). However, whereas Mdivi-1 improved mitochondrial functions and reduced cell death, P259, the inhibitor of physiological fission was injurious, causing a further reduction in mitochondrial membrane potential and a higher mitochondrial ROS production in astrocytes activated by TNFa, IL-1a and C1q (Fig. 5i, j). Together, we found 
that a selective interruption of physiological mitochondrial fission is injurious to the astrocytes. Furthermore, our data indicate that induction of the pro-inflammatory phenotype in microglia or in astrocytes is dependent, at least in part, on Drp1/Fis1-mediated excessive mitochondrial fission and mitochondrial dysfunction and that the propagation of the proinflammatory signal from activated microglia to naïve or primed (R6/2 or WT) is similarly a Drp1/Fis1-dependent process.

\section{Drp1/Fis1-dependent mitochondrial fission increases the ratio of dysfunctional /functional mitochondria released from mouse, rat and human microglia}

Mitochondria can be released from multiple cell types ${ }^{2,27}$. We therefore posited that microglia may release mitochondria or their fragments into the extracellular milieu as physical vessels to propagate neuroinflammatory signal. In support of this notion, we identified functional mitochondria with preserved inner membrane potential and ability to generate ATP in the media of cultured naïve primary microglia (Fig. 6b). By contrast, media of primary microglia activated with LPS/nigericin contained $~ 50 \%$ fewer functional mitochondria (as evidenced by reduced mitochondrial membrane potential and ATP levels in the extracellular mitochondria, Fig. 6b), but more than two-fold greater extracellular mitochondrial protein content, as compared to media from naïve primary microglia (as evidenced by Western blot analysis for mitochondrial proteins VDAC and TOM20; Fig. 6c, left). P110 treatment of LPS/nigericin-activated primary rat microglia did not significantly affect the amount of released mitochondria (Fig. 6c). However, P110 treatment of the microglia greatly reduced the loss cytochrome $\mathrm{c}$, a small $(12 \mathrm{kDa})$ protein compartmentalized within the mitochondrial inter-membrane space and a marker for mitochondrial damage ${ }^{28}$ from the mitochondria released to the cultured media (Fig. 6c). Consistent with improved mitochondrial structure of the extracellular mitochondria, P110 treatment also inhibited the loss of inner membrane potential, and improved ATP production by these extracellular mitochondria, indicative with improved mitochondrial integrity (Fig. $6 b$ ). We can attribute the JC-1 and ATP levels measured in the microglial-conditioned media to the extracellular mitochondria, as filtering the media with 0.2 micron filter ${ }^{2}$; (Fig. 6a) resulted in loss of both JC-1 signal and ATP (Fig. 6b; Mito $\Delta$ ).

We next used microglia of R6/2 mice or BV2 microglia activated by Q73 expression (to model HD) or BV2 microglia expressing mutant SOD1 (to model ALS), and found that all these microglia also released functional mitochondria into the cultured media under basal conditions, but the integrity of the released mitochondria, as measured by mitochondrial membrane potential and ATP production, was lower relative to their corresponding controls (Fig. 6d and e). The amounts of functional extracellular mitochondria were lower if the microglia were activated by expressing mutant huntingtin protein (in microglia derived from R6/2 mice), treated without or with LPS (Fig. 6d) or expressing Q73 or mutant SOD protein (Fig. 6e). As before, the extent of the dysfunction of these released mitochondria was greatly blunted if the cells were treated with P110 (Fig. 6d, e). Importantly, we find that a model of human microglia (differentiated from peripheral blood mononuclear cells ${ }^{29}$ ) also released functional mitochondria under basal conditions that produced ATP, and that activation of these human microglia with LPS/nigericin greatly reduced the integrity of the expelled mitochondria (Fig. 6f); as before, treatment of the LPS-activated human microglia with P110 
improved the integrity and function of the released mitochondria (Fig. 6f). Together, our data show that inhibition of Drp1/Fis1-mediated mitochondrial fission by P110 reduced the dysfunction of the mitochondria released from activated microglia, but not the total amount of mitochondria or mitochondrial proteins released into the extracellular media.

\section{Dysfunctional extracellular microglial mitochondria trigger the release of dysfunctional mitochondria from naïve astrocytes}

As reported before ${ }^{2}$, astrocytes also released functional mitochondria that can produce ATP (Fig. 6g, h, Con vs. Mito $\Delta$ ) and astrocytes activated by conditioned media of LPS/nigericinmicroglia released more damaged mitochondria, as evidenced by $50 \%$ lower mitochondrial membrane integrity and 50\% lower ATP levels (Fig. 6g). The released astrocytic mitochondria exhibited a substantial loss of cytochrome c (Fig. 6h), suggesting damage to the outer membrane of the extracellular mitochondria. As above, the release of these dysfunctional mitochondria from the astrocytes was greatly blunted if the microglia were activated in the presence of P110 (Fig. 6g, h). (Again, because the half-life of P110 is very short, P110 did not exert its affect directly on the astrocytes.) Structural abnormalities of the released astrocytic mitochondria, as evidenced by electron microscopy of the extracellular mitochondria, included reduced cristae structure and breaks in the mitochondrial outer membrane (arrows, Fig. 6i, middle panels $v s$. left panels), which were partially corrected when the mitochondria were isolated from astrocytes treated with conditioned media of LPS/nigericin-treated primary microglial cells co-treated with P110 (Fig. 6i, right vs. middle panels). These data reveal a role for excessive/pathological mitochondrial fission in the genesis and delivery of dysfunctional mitochondria to the extracellular milieu.

To confirm that astrocytes directly release mitochondria, we examined cultured media of astrocytes activated by a mixture of the proinflammatory cytokines, TNFa, IL1a and C1q. This cytokine activation of the astrocytes reduced the function of the extracellular mitochondria by $\sim 50 \%$ relative to those released from naïve (control) astrocytes (Fig. 6j). Dysfunction of the extracellular astrocytic mitochondria was greatly blunted by treatment with P110, the inhibitor of Drp1/Fis1-mediated fission, or with Mdivi-1, an inhibitor of Drp1's catalytic activity ${ }^{30}$. In contrast, here, too, there was no benefit for treatment with P259, a peptide inhibitor of Drp1 interaction of mitochondrial Mff that inhibits physiological but not pathological fission ${ }^{26}$ (Fig. 6j). As the amount mitochondria in the cultured media is unaffected if the stressed cells are treated with the cytoprotective peptide, P110, or the cell damaging peptide, P259 (see LDH release Fig. 5k and Supplementary Fig. 4c and Fig. 6c, h and j), mitochondrial content in the media is unlikely related to the extent of cell death. Examining the cytochrome $\mathrm{c}$ content of the extracellular mitochondria confirmed that the integrity of mitochondrial released from activated astrocytes treated with P110 and Mdivi-1 was better relative to those released from activated astrocytes treated with P259 or vehicle (Fig. 6k), indicating the selective role of Drp1/Fis1-mediated fission in this process.

\section{Extracellular dysfunctional mitochondria propagate injury from microglia to astrocytes to neurons whereas extracellular functional mitochondria are neuroprotective}

As discussed above, much is known about the primary function of all glial cells in protecting and providing essential structural and metabolic support for neurons ${ }^{31}$. Yet, we found that 
transfer of conditioned media of microglia-activated astrocytes (ACM; Fig. 7) or direct transfer of activated microglial conditioned media to cultured primary neurons (Supplementary Fig. 4a) were sufficient to evoke neuronal damage. ATP production and mitochondrial inner membrane potential decreased in these neuronal cells, and production of neurotoxic ROS (Fig. 7a, b). Propagation of the injurious stimulus from the microglia to astrocytes, and from astrocytes to neurons, was consistent whether microglia were activated non-specifically with LPS (Fig. 7a,b) or by recapitulation of genetic neurodegenerative diseases by expressing long poly-glutamine (Q73, as a HD models) or G93A SOD1 (ALS model) (Fig. 7c). Extracellular fragmented mitochondria, but not free mitochondrial DNA ${ }^{32}$, were the major mediators of the major neurotoxic signal released from activated astrocytes: filtering out mitochondrial particles from activated astrocytic-conditioned media (Mito $\Delta$ ) attenuated mitochondrial dysfunction in neurons as measured by neuronal mitochondrial respiration (Fig. 7b) and substantially decreased neuronal cell death (Fig. 7d), whereas enzymatic degradation of free mitochondrial DNA, a known contributor to cytotoxicity ${ }^{33}$, using DNase treatment of the conditioned media had only a small salutary effect (Fig. 7d). Finally, addition of enriched preparation of extracellular astrocytic mitochondrial particulates to cultured neurons without soluble material from the conditioned media caused a-fivefold increased neuronal cell death, which was significantly reduced by P110 treatment of the primary microglia that propagated the injurious stimulus to the neurons via the astrocytic mitochondria (Fig. 7e). Therefore, extracellular mitochondria alone propagate the injury from the activated microglia to the astrocytes and from the astrocytes to the neurons. We also confirmed previous observation by Hayakawa and collegues ${ }^{2}$ that intact extracellular astrocytic mitochondria provide neuroprotection; removal of the mitochondria from the naïve or P110-protected astrocytic-conditioned doubled neuronal cell death (Supplementary Fig. 4b; compare Mito $\Delta$ to -).

The indirect propagation of the neurotoxic signal from the microglia to the astrocytes and subsequently to the neuron was not due to transient expression of neurotoxic proteins in the microglia. Microglia isolated from R6/2 Huntington's disease mouse model also propagated the mitochondrial dysfunction and neuronal death through naïve WT astrocytes (Fig 8a) or through R6/2-derived and likely partially activated astrocytes (Fig. 8b); we observed more than five-fold higher ROS production in the neuronal mitochondria and a 50\% more neuronal cell death when the injurious stimulus from the microglia was propagated through R6/2-derived astrocytes as compared with littermate WT astrocytes (Fig. 8b). Again, a greater propagation of neuronal injury was observed when the microglia were also cultured in the presence of LPS and P110 treatment of the microglia (R6/2 or WT) was sufficient to greatly blunt the propagation of injury through the astrocytes (R6/2 or WT) to the primary neurons (Fig. 8a, b).

\section{Discussion}

The relationship between mitochondria and eukaryotes has been critical for the success of metazoan life on earth. Cell colonization by ancestral a-proteobacteria over a billion years ago conferred advantages in energy production and oxygen utilization. However, host cells had to recognize and protect their increasingly essential endosymbionts while simultaneously identifying and repelling phylogenetically-related pathogenic bacterial 
invaders. Consequently, mitochondria became immunologically privileged. Nevertheless, misidentification of extracellular mitochondrial DNA, damaged mitochondria or other damage-associated molecular patterns (DAMPs) as bacterial can trigger innate (sterile) immune mechanisms that, in turn, contribute to mitochondrial dysfunction and further propagation of the pathology in acute and chronic inflammatory diseases ${ }^{32}$. The mechanisms underlying mitochondrial loss of immune privileged state and their transformation into mediators of pathological entities in neurodegenerative disease are unclear.

\section{Loss of immune privileged state of mitochondria in models of neurodegenerative diseases.}

Our results here show that loss of the immune-privileged state of extracellular damaged mitochondria correlates with increased release of damaged mitochondria from the microglia and that damaged extracellular mitochondria directly contribute to disease propagation by acting as effectors of innate immune response, targeting adjacent astrocytes and neurons. Our data here also show that in addition to the direct effect we previously reported on neurons harboring neurotoxic proteins ${ }^{12,16,18,20}$, the Drp1/Fis1 inhibitory peptide, P110, reduces mitochondrial fission and consequent release of damaged mitochondria from the microglia, thus inhibiting astrocytes' activation and protecting neurons from innate immune attack. Increased Drp1/Fis1-mediated mitochondrial fission in activated microglia triggers formation of fragmented and damaged mitochondria that are released from these cells, thus inducing innate immune response. Therefore, although clinical and experimental studies have identified fragmented mitochondria in biofluids of subarachnoid hemorrhage and stroke patients ${ }^{2,34-36}$ suggesting their presence in the extracellular space is a biomarker for neurodegeneration and disease severity ${ }^{37,38}$, our data here show a causal role of dysfunctional extracellular mitochondria in propagating neurodegenerative signals from microglia.

Innate immune response in neurodegenerative diseases begins early in the pathogenesis of these diseases and is associated with little or no infiltration of blood-derived immune cells into the brain ${ }^{39}$. It is the brain-resident cells, the microglia and astrocytes, that trigger this sterile immune response, thus contributing to neuronal dysfunction and degeneration.

Although mice exclusively expressing mutant huntingtin in microglia, for example, show increased innate immune response and increased neuronal cell death ${ }^{22}$, activated microglia alone are unlikely to cause major neurodegenerative disease $e^{4,5,40}$. Rather our data suggest that a relay of glia-to-neurons-to-glia signaling (Supplementary Fig. 4d) plays an important role in neurodegeneration. Feeding into the vicious cycle, the death of neurons, induced by the neurotoxic proteins, generates further cellular debris and these debris (DAMPs), together with dysfunctional mitochondria released from microglia expressing neurotoxic proteins, exacerbate astrocytes activation and chronic pathogenic inflammation. Thus, neuronal cell death and the final phenotype of the disease occurs via innate immune response activation as well as via direct effect of neurotoxic protein-induced neuronal cell death. 


\section{Activation of innate immune response and neurotoxic protein-induced neuronal cell death in models of neurodegenerative diseases are both dependent on Drp1/Fis1-mediated excessive mitochondrial fragmentation.}

We have previously shown that expression of neurotoxic proteins in cultured neurons and the resulting cell death can be greatly blunted by inhibiting Drp1/Fis1-mediated mitochondrial fragmentation using P110 peptide ${ }^{16,20}$. Here, we show that the innate immune response in several models of neurodegenerative diseases is also dependent on pathological Drp1/Fis1dependent mitochondrial fission in the microglia and the resulting release of dysfunctional mitochondria to the extracellular milieu. However, the mechanisms by which mitochondria are released to the extracellular space are still under investigation. For example, mitochondrial transfer may occur via release in extracellular vesicles $(\mathrm{EVs})^{41}$, structures that are $50-1,000 \mathrm{~nm}$ in diameter ${ }^{42}$, and astrocytes and microglia release $\mathrm{EVs}^{43-45}$. But other mechanisms, including direct exocytosis may contribute to this release ${ }^{46}$. Also not known yet is what is the minimal amount of damaged mitochondria required for the propagation of neuronal cell death and whether transfer of functional mitochondria between microglia and astrocytes and from glia to neurons has a role under physiological conditions. What we do know, however, is that extracellular mitochondria are critical in mediating this cell to cell propagation of pathological signaling.

\section{The ratio between damaged mitochondria and functional mitochondria in the extracellular milieu governs the outcome to neurons.}

Although extracellular damaged mitochondria are injurious, transfer of functional mitochondria is protective (Supplementary Figure 4), as has been previously shown for example in a mouse model of acute lung injury ${ }^{47}$ and in a model of stroke ${ }^{2}$. Whether the extracellular damaged mitochondria enter the neurons, as has been suggested for the functional mitochondria in the previous study ${ }^{2}$, has not yet been determined. The novel finding of our study is that it is not the amount of the extracellular mitochondria, but rather the ratio between damaged mitochondria and functional mitochondria in the extracellular milieu that governs the outcome to neurons and is determined by the extent of pathological fission in the donor microglia. The structural and functional integrity of the released glial mitochondria were confirmed by measuring respiration, mitochondrial membrane potential, ATP production, content of cytochrome c (indicating mitochondrial outer membrane integrity) and electron microscopy. Although the amount of extracellular mitochondria was unaltered, all these parameters showed improved structure and function of the extracellular mitochondria released from microglia after treatment with P110.

Our data suggest that selective inhibition of pathological mitochondrial fission in the microglia (mediated by Drp1/Fis $1{ }^{16}$ ) without affecting physiological mitochondrial fission (mediated by Drp1/Mff; ${ }^{26}$ ) reduced propagation of neuronal injury by two mechanisms.

First, P110 reduced activation of innate immune response in microglia and in astrocytes and cytokine-mediated neuronal cell death induced by extracellular dysfunctional mitochondria. And second, inhibition of pathological mitochondrial fission by P110 in the donor microglia supported neuronal cell survival by increased ratio of healthy to damaged mitochondria released from the donor cells, thus protecting the neuron. 
Suppression of Drp1/Fis1-mediated mitochondrial fission is a readily translatable approach for interrupting this pathological microglia-to-astrocyte-to-neuron mitochondrial dysfunction, neuroinflammation and neuronal cell death mechanisms and supporting transfer of healthy mitochondria to neurons. However, we envision that any means of normalizing the balance between healthy and damaged mitochondria within the neuronal milieu, as by clearing damaged and fragmented mitochondrial with specific antibodies or by introducing healthy mitochondria could also provide neuronal protection in neurodegenerative diseases.

\section{Methods}

\section{Data reporting}

All procedures were conducted in accordance with the animal care standards of the National Institute of Health and approved by Stanford University's Administrative Panel on Laboratory Animal Care. All chemicals were purchased from Sigma-Aldrich (St Louis, MO) unless stated otherwise.

\section{Peptides}

Drp1/Fis1 peptide inhibitor P110 (derived from Drp149-55-GG-TAT $47-57$ ), Drp1/Mff peptide inhibitor P259 (derived from Drp1 22-31-GG-TAT $47-57$ ) and control peptide TAT (derived from $\mathrm{TAT}_{47-57}$ ) were synthesized by Ontores Biotechnologies (Hangzhou, China) ${ }^{12}$. The purity of peptides was $>90 \%$ measured by RP-HPLC Chromatogram.

\section{Cell lines and transient expression of mutant proteins}

BV2 mouse microglial cells were cultured in either $75 \mathrm{~cm}^{2}$ flasks, 6-well or 24-well dishes containing RPMI medium supplemented with $10 \%$ FBS, 4 mM glutamine, $100 \mathrm{U} / \mathrm{ml}$ penicillin, $10 \mu \mathrm{g} / \mathrm{ml}$ streptomycin and grown in a $5 \% \mathrm{CO}_{2}$ atmosphere at $37{ }^{\circ} \mathrm{C}$. Cultured BV2 microglia were seeded at $5 \times 10^{4}$ cells $/ \mathrm{mL}$ and allowed to attach before transfection with either green fluorescent protein (GFP)-wildtype SOD1 (control) or mutant G93A mutant SOD1 (ALS-like model) or Q23 (control) or Q73 (HD-like model) using the transfection reagent, according to the instructions provided by the manufacturer (Lipofectamine 2000 reagent, Invitrogen) for $48 \mathrm{~h}$. Htt ${ }^{\mathrm{ex}} \mathrm{Q} 23$ and Q73 constructs were obtained from the CHDI Foundation. pF145 pAcGFP1 SOD1G93A and pF141 pAcGFP1 SOD1WT were a gift from Elizabeth Fisher (Addgene plasmid \# 26406/\# 26402) ${ }^{48}$. Transfection efficiency was tested by using a vector containing the GFP gene and reached usually $60 \%$.

\section{Primary neuron cultures}

Primary neuron cultures were prepared from cerebral cortices of embryonic day (E)17 Sprague-Dawley rat embryos or E17 C57/BL6 mice. In brief, cortices were dissected and dissociated using papain dissociation system (Worthington Biochemical Corporation, Cat no\# LK003150). Cells were spread at 20,000/well of a 96 well plate coated with poly-Dlysine (Sigma, Cat no\# P7886) for cell health assays; for seahorse experiments, $1 \times 10^{5}$ cells/ well were seeded in XF 24-well cell culture microplate and cultured in Neurobasal medium (Invitrogen, Cat no\# 21103-049) supplemented with B-27 (Invitrogen, Cat no\# 17504044) containing $25 \mathrm{mM}$ glucose, $4 \mathrm{mM}$ glutamine, $1 \mathrm{mM}$ sodium pyruvate, and 5\% FBS. At $24 \mathrm{~h}$ after seeding, the medium was changed to Neurobasal medium supplemented with B-27 and 
$0.5 \mathrm{mM}$ glutamine. Cells were cultured at $37^{\circ} \mathrm{C}$ in a humidified chamber of $95 \%$ air and $5 \%$ $\mathrm{CO}_{2}$. Cultures were used for experiments from 7 to 10 days after seeding.

\section{Primary astrocyte and microglia cultures from WT rats and mice and from R6/2 mice and their littermates.}

Primary astrocyte cultures were prepared from cerebral cortices of 2-day-old neonatal Sprague-Dawley rats or P1/2 C57BL/6 J mice or P1/2 R6/2 or littermates (B6CBA-TgN (HD exon1)62; JAX stock number: 006494). In brief, dissociated cortical cells were suspended in DMEM/F12 50/50 (Life Technology, Cat no\# 11320-033) containing $25 \mathrm{mM}$ glucose, $4 \mathrm{mM}$ glutamine, $1 \mathrm{mM}$ sodium pyruvate, and 10\% FBS and plated on uncoated 25 $\mathrm{cm}^{2}$ flasks at a density of $6 \times 10^{5}$ cells $\mathrm{cm}^{2}$. Monolayers of type 1 astrocytes were obtained 12-14 days after plating. Cultures were gently shaken, and floating cells (microglia) were collected, resulting in more than $95 \%$ pure culture of astrocytes. Astrocytes were dissociated by trypsinization and then reseeded at $3-5 \times 10^{5}$ cells $/ \mathrm{cm}^{2}$ or $1.5 \times 10^{5}$ cells $/$ well in 24 well or $1.5 \times 10^{6}$ cells/well in 6 well or 1200 cells/well in 96 well, PDL-coated culture vessels in DMEM F12 50/50 containing 15\% FBS and 1\% penicillin/streptomycin. For seahorse experiments, astrocytes were seeded at $4 \times 10^{4}$ cells/well in XF 24-well cell culture microplate. At the start of experiment, media was changed to DMEM without serum and antibiotics. Astrocytic-conditioned media (ACM) were collected $24 \mathrm{~h}$ later and cleared of cellular debris with centrifugation at $1,000 \mathrm{~g}$ for $10 \mathrm{~min}$.

Isolated microglia cells collected after shaking mixed glial culture were seeded at primary microglia were plated at $2.4 \times 10^{6}$ cells/well in 6 -well plates or $3 \times 10^{5}$ cells/well in 24 -well plates or 2500 cells/well in 96 well incubated with serum-free antibiotic free macrophage medium (Invitrogen, Cat no\# 12065074) for 24 hours before switching to DMEM without serum and antibiotics at the start of various experiments. Collected microglia conditioned media (MCM) was then centrifuged at $1,000 \mathrm{~g}$ for $10 \mathrm{~min}$ for removal of cell debris. For seahorse experiments, microglia were seeded at $1.25 \times 10^{5}$ cells/well in XF 24-well cell culture microplate.

\section{Primary human monocyte-induced microglia}

Primary human monocyte-derived microglia like (MDMi) cells were generated as previously described $^{29,49}$. In brief, peripheral blood mononuclear cells from de-identified healthy donors (35-65 years old) were obtained from the Stanford Blood center. Samples were diluted with $20 \mathrm{ml}$ PBS and layered onto $10 \mathrm{ml}$ of Ficoll-Paque (GE Healthcare, Cat no \# 17144002) using a Pasteur pipette. Tubes were centrifuged at $1500 \mathrm{rpm}$ for $25 \mathrm{~min}$ without brake. Mononuclear cell layer was isolated, resuspended in 50ml of PBS, and centrifuged at $1500 \mathrm{rpm}$ for $10 \mathrm{~min}$. Monocytes were then isolated using a Pan Monocyte Human Isolation Kit (MACS; Miltenyi biotech, Cat no \# 130-096-537) and plated at a confluency of $10 \times 10^{6}$ cells per $10 \mathrm{~cm}$ plate. Cells were differentiated for 10 days at $37^{\circ} \mathrm{C} 5 \mathrm{CO}_{2}$ in Roswell Park Memorial Institute (RPMI) media 1640 GlutaMAX (ThermoFisher Scientific, Cat no \# 61870036) and supplemented with 10\% FBS, 1\% penicillin-streptomycin, 10ng/ml M-CSF, $10 \mathrm{ng} / \mathrm{ml}$ GM-CSF, $10 \mathrm{ng} / \mathrm{ml} \mathrm{NGF-} \beta$, $100 \mathrm{ng} / \mathrm{ml} \mathrm{CCL2}$, and 100ng/ml IL-34 (all BioLegend CNS, Inc). All the experiments were performed in serum free antibiotic free RPMI. 


\section{oA $\beta$ preparation}

Synthetic $A \beta_{1-42}$ was purchased from Anaspec (Fremont, CA, USA, Cat no \# AS-25381) and prepared according to protocols described elsewhere ${ }^{18}$. Briefly, lyophilized $A \beta_{1-42}$ peptides were dissolved in 1,1,1,3,3,3-hexafluoro-2-propanol (HFIP, Sigma Aldrich Cat no \# 325244) and aliquoted into polypropylene micro-centrifuge tubes. HFIP was evaporated and the resulting peptide films were stored at $-80^{\circ} \mathrm{C}$. Before use, these peptide films were reconstituted at a concentration of $1 \mathrm{mM}$ in dimethylsulphoxide (DMSO, Sigma Aldrich Cat no \# D8418), then subsequently diluted to $100 \mu \mathrm{M}$ with ice-cold DMEM (phenol red-free, Invitrogen Cat no \# 21063029) and incubated for $24 \mathrm{~h}$ at $4^{\circ} \mathrm{C}$ to facilitate the formation of oligomers. Resultant peptides were stored at $-80^{\circ} \mathrm{C}$ until use.

\section{Cell health assays}

Cell viability assays-Cell viability was determined measuring the activity of lactate dehydrogenase (LDH) released (Cytotoxicity Detection KitPLUS (LDH), Sigma Aldrich Cat no \# 4744934001) according to manufacturers' instructions. Briefly, at the end of the incubation, supernatants of cultured media were collected and centrifuged at $1000 \times \mathrm{g}$ to remove any residual cell debris. The reaction mixture was added to the cell-free supernatant, and color development was measured spectrophotometrically at $492 \mathrm{~nm}$ with a reference wavelength at $610 \mathrm{~nm}$. Primary astrocytes and primary neurons were treated with different stimuli for 48 or 24 hours respectively before analysis.

Mitochondrial ROS production-To determine mitochondrial ROS production, cells were treated with $5 \mu \mathrm{M}$ MitoSOX ${ }^{\mathrm{TM}}$ Red mitochondrial superoxide indicator (Invitrogen, Cat no \# M36008) and $10 \mu \mathrm{g} / \mathrm{ml}$ of nuclear staining dye Hoechst-33342 (Sigma-Aldrich, Cat no \# B 2261) at the end of the experiment for $15 \mathrm{~min}$ at $37^{\circ} \mathrm{C}$ according to manufacturer's protocol. Fluorescence was recorded for MitoSOX (Ex 510nm and Em $580 \mathrm{~nm}$ ) and Hoechst-33342 (Ex $350 \mathrm{~nm}$ and Em $461 \mathrm{~nm}$ ) in SpectraMax M2e (Molecular devices). MitoSOX fluorescence was normalized with Hoechst reading. Mitochondrial ROS levels in primary microglia were analyzed either at 16 hours in HD model and ALS model, 21 hours in LPS/nigericin model or 24 hours in AD model after individual stimuli. Mitochondrial ROS levels in primary astrocytes were analyzed either at 6 hours in HD and ALS models or 24 hours for all other stimuli. Mitochondrial ROS levels in primary neurons were analyzed either at 24 hours after stimuli.

ATP measurement-Relative intracellular or extracellular ATP levels were determined using ATP-based CellTiter-Glo Luminescent Cell Viability kit (Promega, Cat no \# G7570), which can perform cell lysis and generate a luminescent signal proportional to the amount of ATP present. In brief for intracellular ATP levels, opaque-walled 96-well plates with cell lysate $(50 \mu \mathrm{l})$ were prepared. An equal volume of the single-one-step reagent provided by the kit was added to each well and incubated for $30 \mathrm{~min}$ at room temperature. For measuring ATP content in extracellular mitochondria, cell supernatant was cleared of cellular debris by centrifugation at $1000 \mathrm{~g}$ for $10 \mathrm{~min}$ and then centrifuged $13000 \mathrm{~g}$ for $25 \mathrm{~min}$ followed by a wash with $1 \mathrm{ml}$ of PBS. The pellet was then resuspended in the 50ul of serum free phenol free DMEM or PBS before an equal volume of the single-one-step reagent provided by the kit with incubation for $30 \mathrm{~min}$ at room temperature. ATP content was measured using a 
luminescent plate reader SpectraMax M2e (Molecular devices). Mitochondrial ATP levels in primary microglia were analyzed either at 16 hours in HD model and ALS model, 21 hours in LPS/nigericin model or 24 hours in AD model after individual stimuli. Mitochondrial ATP levels in primary astrocytes were analyzed either at 6 hours in HD and ALS models or 24 hours for all other stimuli. Mitochondrial ATP levels in primary neurons were analyzed either at 24 hours after stimuli. For extracellular mitochondria experiments, ATP levels in the $13,000 \mathrm{~g}$ pellet were measured after end of total stimulation of 24 hours.

Mitochondria membrane potential measurement-To monitor mitochondrial health, JC-1 dye (Invitrogen, Cat no \# T-3168) was used to assess mitochondrial membrane potential. In brief, cell supernatant was cleared of cellular debris by centrifugation at $1000 \mathrm{~g}$ for $10 \mathrm{~min}$ at the end of the experiment ( 24 hours). The cell supernatant was then loaded with $1 \mu \mathrm{M} \mathrm{JC} 1$ dye for $30 \mathrm{~min}$ at $37^{\circ} \mathrm{C}$. JC1 dye exhibits potential-dependent accumulation in mitochondria, indicated by fluorescence emission shift from green (Ex $485 \mathrm{~nm} / \mathrm{Em} 516$ $\mathrm{nm})$ to red (Ex $579 \mathrm{~nm} / \mathrm{Em} 599 \mathrm{~nm}$ ). Mitochondrial membrane potential was determined by the fluorescent ratio using SpectraMax M2e (Molecular devices). Cells were incubated with tetra-methyl-rhodamine methyl ester (TMRM, Invitrogen, Cat no \# T668) in HBSS (Hank's balanced salt solution, Invitrogen, Cat no \# 14170112) for $30 \mathrm{~min}$ at $37^{\circ} \mathrm{C}$, as per the manufacture's protocol, and the fluorescence was analyzed using SpectraMax M2e (Molecular devices, using excitation at $360 \mathrm{~nm}$ and emission at $460 \mathrm{~nm}$ ). Mitochondrial membrane levels in primary microglia and primary astrocytes were analyzed at 24 hours after individual stimuli.

Bioenergetic profiles-Cells were plated in a Seahorse XF24 Cell Culture Microplate (Agilent). All seahorse experiments in microglia, astrocytes and neurons were performed at 24 hours after individual stimuli. At the end of treatment cells were washed twice with Agilent Seahorse XF Media (Agilent) supplemented with $1 \mathrm{mM}$ pyruvate, $2 \mathrm{mM} \mathrm{L-}$ glutamine, and $2 \mathrm{mM}$ D-glucose; a final volume of $525 \mu \mathrm{l}$ was placed in each well. Cells were then incubated in a $0 \% \mathrm{CO}_{2}$ chamber at $37{ }^{\circ} \mathrm{C}$ for $1 \mathrm{~h}$ before being placed into a Seahorse XFe24 Analyzer (Agilent). For oxygen consumption rate (OCR) and (extracellular acidification rate) ECAR experiments, cells were treated with $1 \mu \mathrm{M}$ oligomycin, $2 \mu \mathrm{M}$ carbonyl cyanide p-trifluoromethoxy phenylhydrazone (FCCP), and $0.5 \mu \mathrm{M}$ rotenone/ antimycin. A total of three OCR and $\mathrm{pH}$ measurements were taken after each compound was administered.

Immunocytochemistry and immunohistochemistry-Immunohistochemistry was performed as described previously ${ }^{12}$. Mitochondrial structure analysis in microglia was performed after 24 hours of the initial stimuli. For astrocytes, microglial conditioned media (MCM) was added to astrocytes for 24 hours before analysis. Cells cultured on 8-well chamber slides were washed with cold PBS, fixed in $4 \%$ formaldehyde, and permeabilized with $0.1 \%$ Triton X-100. After incubation with $2 \%$ normal goat serum (to block nonspecific staining), fixed cells were incubated overnight at $4^{\circ} \mathrm{C}$ with anti-TOM20 primary antibody (1:500) (Santa Cruz, USA). Cells were washed with PBS and incubated for 60 minutes with FITC-conjugated goat anti-rabbit IgG (1:500 dilution). The cells were then washed gently with PBS and counterstained with Hoechst 33342 (1:10,000 dilution, Molecular Probes) to 
visualize nuclei. The coverslips were mounted with Slowfade-antifade reagent (Invitrogen). Parameters of mitochondrial morphology were further quantified with Fiji (ImageJ) as described before ${ }^{50}$. Detailed workflow is represented in Supplementary figure 5.

For the immunohistochemistry studies, mice described in our previous publications ${ }^{12,18,19}$. Adult B6SJL Tg (SOD1G93A) 1 Gur/J male mice with a high copy number of the mutant allele and their WT littermates at 120 days, 5XFAD transgenic male mice and their littermates at 6 months, and Hemizygous male R6/2 HD mice and their WT littermates at 12 weeks were analyzed. In brief, mice were sacrificed, tissue dissected, and fixed in $4 \%$ paraformaldehyde in $0.1 \mathrm{M}$ phosphate buffer, $\mathrm{pH}$ 7.4. Tissues were then paraffin-embedded, and sections were used for immunohistochemical staining of GFAP and Iba-1 (done by Histo-tec Laboratory, Hayward, CA). The images were acquired using an All-in-One Fluorescence Microscope BZ-X700 (Keyence). The level of GFAP or Iba-1 immunoreactivity was measured by using mean optical density of DAB staining. The white balance of all images was standardized to eliminate color bias.

\section{Isolation of mitochondria-enriched fraction and lysate preparation for western} blot analysis-Cells were washed with cold phosphate-buffered saline (PBS) at pH 7.4 and scraped off using mannitol-sucrose (MS) buffer containing $210 \mathrm{mM}$ mannitol, $70 \mathrm{mM}$ sucrose, $5 \mathrm{mM}$ MOPS (3-(N-morpholino) propanesulfonic acid), $1 \mathrm{mM}$ EDTA, and protease inhibitor cocktail, $\mathrm{pH}$ 7.4. The collected cells were passed through a 27 -gauge 1/2-inch needle for lysis, followed by centrifugation at $800 \times \mathrm{g}$ to pellet nuclei. The post-nuclear supernatant was further centrifuged at $10,000 \times \mathrm{g}$ for $20 \mathrm{~min}$ to collect a mitochondriaenriched fraction as previously described before ${ }^{12}$.

Electron microscopy-Pellets from astrocyte-conditioned media (precleared cell supernatant at $1000 \mathrm{~g}$ for $10 \mathrm{~min}$ followed by $13000 \mathrm{~g}$ for $15 \mathrm{~min}$ ) were fixed in $2.0 \%$ glutaraldehyde in $0.1 \mathrm{M}$ sodium cacodylate buffer, $\mathrm{pH} 7.4$ for one hour at room temperature on a rocker. They were rinsed in cacodylate buffer, gently scraped and pelleted and postfixed in $1.0 \%$ osmium tetroxide in cacodylate buffer for one hour on ice. They were rinsed in buffer and stabilized with a small amount of $2 \%$ agarose in PBS to hold them together. They were then dehydrated through a graded series of ethanol to $100 \%$, followed by propylene oxide, 100\%. They were infiltrated with Epon resin (Ted Pella, Redding, CA) in a 1:1 solution of Epon: propylene oxide overnight on a rocker at room temperature. The following day they were placed in fresh Epon for several hours and then embedded in Epon overnight at $60^{\circ} \mathrm{C}$. Samples were observed in a JEOL 1230 transmission electron microscope at 80 $\mathrm{kV}$, and photographs were taken using a Gatan Multiscan 791 digital camera.

ELISA-Mouse/rat IL-6 (Cat no \# 5017218/50-183-46), mouse/rat IL-1a (Cat no \# 501125107/5018351), mouse/rat TNFa (Cat no \# 50-112-8954/5017347), IL-1 $\beta$ (Cat no \# 501129749/501125471) ELISA kits (eBiosciences) were used to quantify cytokine levels in mouse tissue and cell supernatant according to manufactures protocols.

RNA isolation and gene expression analysis-RNA isolation was performed using GenElute ${ }^{\mathrm{TM}}$ Mammalian Total RNA Miniprep Kit (Sigma Aldrich, Cat no \# RTN350) according to manufacturer's protocols. RNA concentration was measured using a Nanodrop 
(ND -1000; NanoDrop Technologies, Rockland, DE, USA) and RNA integrity was assessed using a Bioanalyzer (2100; Agilent Technologies, Palo Alto, CA, USA). cDNA synthesis was performed using the Quantitect reverse transcription kit (Qiagen, Cat no \# 205313) according to manufacturer's instructions, with a minimal input of 200 ng total RNA. Quantitative PCR (qPCR) was performed using the 7300 Real Time PCR system (Applied Biosystems, Foster City, USA) using the equivalent cDNA amount of 1-2 ng total RNA used in cDNA synthesis. SYBRgreen master mix (Applied Biosystems, Cat no \#43-127-04) and a $2 \mathrm{pmol} / \mathrm{ml} \mathrm{mix}$ of forward and reverse primer sequences were used for 40 cycles of target gene amplification. The primers used in this study are $18 S r R N A$ :

5'CTTAGAGGGACAAGTGGCG 3' ACGCTGAGCCAGTCAGTGTA; ARG1: 5'ATGGAAGAGACCTTCAGCTAC 3' GCTGTCTTCCCAAGAGTTGGG; $\boldsymbol{A} \boldsymbol{X \boldsymbol { L }}$ : 5'ATGGCCGACATTGCCAGTG 3' CGGTAGTAATCCCCGTTGTAGA; $\boldsymbol{C 1 Q A \text { : }}$

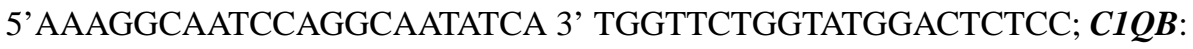
5' AAGATCCAGAAACACAAGTCCCT 3' CCTCCTCACCATCAAATGTTGG; C3: 5'CCAGCTCCCCATTAGCTCTG 3' GCACTTGCCTCTTTAGGAAGTC; $\boldsymbol{C A T}$ : 5'TAAGACTGACCAGGGCA 3' CAAACCTTGGTGAGATCGAA; CCL4: 5'TTCCTGCTGTTTCTCTTACACCT 3' CTGTCTGCCTCTTTTGGTCAG; CD14: 5'GGCGCTCCGAGTTGTGACT 3' TACCTGCTTCAGCCCAGTGA; CD86: 5'TGTTTCCGTGGAGACGCAAG 3' TTGAGCCTTTGTAAATGGGCA; $\boldsymbol{G A P D H}$ : 5'CATCACTGCCACCCAGAAGACTG 3' ATGCCAGTGAGCTTCCCGTTCAG; GAS6: 5'TGCTGGCTTCCGAGTCTTC 3' CGGGGTCGTTCTCGAACAC; GFAP: 5'CCCTGGCTCGTGTGGATTT 3' GACCGATACCACTCCTCTGTC; ILl10: 5'ATTTGAATTCCCTGGGTGAGAAG 3' CACAGGGGAGAAATCGATGACA; ILl3: 5'AGACCAGACTCCCCTGTGCA 3' TGGGTCCTGTAGATGGCATTG; IL33: 5'TGAGACTCCGTTCTGGCCTC 3' CTCTTCATGCTTGGTACCCGAT; $\boldsymbol{I L 4}$ : 5'AGATGGATGTGCCAAACGTCCTCA 3' AATATGCGAAGCACCTTGGAAGCC; ITGAM: 5' ATGGACGCTGATGGCAATACC 3' TCCCCATTCACGTCTCCCA; ITGB2: 5'CACTGTCTCAGTTGTGTACCAAG 3' GCTCTGGTGTATCACAGCGAA; ITGB3: 5'GAGCCCATTTTCTTCTCCCG 3' GCAACACCATGAATCCATCCC; $\boldsymbol{M E G F ~ 1 0 : ~}$ 5'GAAGACCCCAACGTATGCAG 3' CGGTGCAGCTTGTGTAGTAGA; MERTK: 5'CAGGGCCTTTACCAGGGAGA 3' TGTGTGCTGGATGTGATCTTC; SOD2: 5'ACAGGCCTTATTCCACTGCT 3' CAGCATAACGATCGTGGTTT; NOX4: 5'TCTGGCTCT-CCATGAATGTC 3' CTGCTTGGAACCTTCTGTGA; NFE2L2: 5'TCTCCTCGCTGGAAAAAGAA 3' AATGTGCTGGCTGTGCTTTA; PPARGC1A: 5'TGGAGTGACATCGAGTGTGCT 3' GAGTCCACCCAGAAAGCTGT; TFAM:

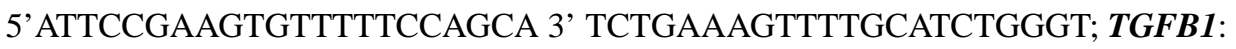
5'GGAGAGCCCTGGATACCAAC 3' CAACCCAGGTCCTTCCTAAA; TTR: 5'TTGCCTCGCTGGACTGGTA 3' TTACAGCCACGTCTACAGCAG; VEGFA: 5'GGAGATCCTTCGAGGAGCACTT 3' GGCGATTTAGCAGCAGATATAAGAA; ACTB: 5'TGGAATCCTGTGGCATCCATGAAAC 3' TAAAACGCAGCTCAGTAACAGTCCG.

Microfluidic qPCR (pooled cell samples)-Total RNA was extracted from cells using the RNeasy Plus kit (Qiagen, Cat no \# 74136) and cDNA synthesis performed using the SuperScript VILO cDNA synthesis kit (Invitrogen) according to supplier protocols. Microfluidic qPCR was conducted using $1.25 \mu \mathrm{l}$ of each cDNA sample pre-amplified using 
$2.5 \mu \mathrm{l}$ of $2 \times$ Taqman pre-amplification master mix (Applied Biosystems) and $1.25 \mu \mathrm{l}$ of the primer pool $(0.2 \mathrm{pmol}$ each primer per $\mu \mathrm{l}$, - primers to rat transcripts for reactive astrocyte subtype were used as previously described ${ }^{9}$. Pre-amplification was performed $\left(10 \mathrm{~min} 95^{\circ} \mathrm{C}\right.$ denaturation step and 14 cycles of $15 \mathrm{~s}$ at $95^{\circ} \mathrm{C}$ and $4 \mathrm{~min}$ at $60^{\circ} \mathrm{C}$ ) and reaction products were diluted $5 \times$ in TE Buffer (Teknova). Five microliters from a sample mix containing preamplified cDNA and amplification Master mix (20 mM MgCl2, $10 \mathrm{mM}$ dNTPs, FastStart

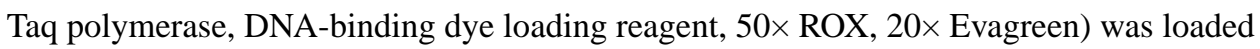
into each sample inlet of a 96.96 Dynamic Array chip (Fluidigm Corporation) and $5 \mu \mathrm{l}$ from an assay mix containing DNA-assay loading reagent, as well as forward and reverse primers (10 pmol $\mu \mathrm{l}-1)$ was loaded into each detector inlet. The chip mixed and loaded in a NanoFlexTM 4-IFC Controller (Fluidigm) and processed in the BioMark Real-Time PCR System (Fluidigm) using a cycling program of $10 \mathrm{~min}$ at $95^{\circ} \mathrm{C}$ followed by 40 cycles of $95^{\circ} \mathrm{C}$ for $15 \mathrm{~s}, 60^{\circ} \mathrm{C}$ for $30 \mathrm{~s}$ and $72^{\circ} \mathrm{C}$ for $30 \mathrm{~s}$. After completion of qPCR, a melting curve of amplified products was determined. Data were collected using BioMark Data Collection Software 2.1.1 build 20090519.0926 (Fluidigm) as the cycle of quantification (Cq), where the fluorescence signal of amplified DNA intersected with background noise. Fluidigm data were corrected for differences in input RNA using the geometric mean of three reference genes ALDH111, GAPDH and Rplp0. Data preprocessing and analysis was completed using Fluidigm Melting Curve Analysis Software 1.1.0 build 20100514.1234 (Fluidigm) and Realtime PCR Analysis Software 2.1.1 build 20090521.1135 (Fluidigm).

Western blot-Protein concentrations were determined using the Bradford assay (Thermo Fisher Scientific). Proteins were resuspended in Laemmli buffer containing 2mercaptoethanol, loaded on SDS-PAGE, and transferred on to nitrocellulose membrane, $0.45 \mu \mathrm{m}$ (Bio-Rad), as before ${ }^{12}$. For extracellular mitochondria analysis, cell supernatant was cleared of cellular debris by centrifugation at $1000 \mathrm{~g}$ for $10 \mathrm{~min}$ and then centrifuged $13000 \mathrm{~g}$ for $25 \mathrm{~min}$ followed by a wash with $1 \mathrm{ml}$ of PBS. The pellet was then resuspended in the 20ul of Laemmli buffer containing 2-mercaptoethanol, loaded on SDS-PAGE, and transferred on to nitrocellulose membrane, $0.45 \mu \mathrm{m}$ (Bio-Rad), as before ${ }^{12}$. Membranes were cut at appropriate molecular weights and then probed with the indicated antibody and visualized by ECL ( $0.225 \mathrm{mM}$ p-coumaric acid; Sigma), $1.25 \mathrm{mM}$ 3-aminophthalhydrazide (Luminol; Fluka) in $1 \mathrm{M}$ Tris $\mathrm{pH}$ 8.5. Scanned images of the exposed X-ray film or images acquired with Azure Biosystems C600 were analyzed with ImageJ to determine relative band intensity (Supplementary Fig 6). Quantification was performed on samples from independent cultures for each condition. The antibodies used in this study are: Anti-ASC (B3) mouse monoclonal antibody (Santa Cruz Biotechnology; sc-514414; 1:200); AntiCOX-2 rabbit polyclonal antibody (Abcam; Ab15191; 1:500); Anti-cytochrome c (7H8.2C12) mouse monoclonal antibody (Abcam; ab13575; 1:1000); Anti-Drp1 (Clone 8/ DLP1 (RUO)) mouse monoclonal antibody (BD Transduction Laboratories; 611113; 1:500); Anti-Enolase-1 rabbit polyclonal antibody (Cell Signaling Technology; 3810; 1:500); AntiGFAP rabbit polyclonal antibody (Abcam; ab7260; 1:500); Anti-Iba-1 goat polyclonal antibody (Abcam; ab5076; 1:1000); Anti-IL1 $\beta$ rabbit polyclonal antibody (Abcam; ab9722; 1:200); Anti-NLRP3 (D4D8T) rabbit monoclonal antibody (Cell Signaling Technology; 15101; 1:200); Anti-p20 (D4) mouse monoclonal antibody (Santa Cruz Biotechnology; sc-398715; 1:100); Anti-phospho SAPK/JNK (Thr183/Tyr185) rabbit polyclonal antibody 
(Cell Signaling Technology; 9251; 1:500); Anti-phospho NFkB p65 (Ser536) (93H1) rabbit monoclonal antibody (Cell Signaling Technology; 3033; 1:500); Anti-phospho p44/42 MAPK (Erk1/2) (Thr202/Tyr204) rabbit polyclonal antibody (Cell Signaling Technology; 9101; 1:500); Anti-phospho-Drp1 (Ser616) rabbit polyclonal antibody (Cell Signaling Technology; 3455; 1:200); Anti-phospho-Drp1 rabbit polyclonal antibody (Ser637) (Cell Signaling Technology; 4867; 1:200); Anti-TOM20 (FL-145) rabbit polyclonal antibody (Santa Cruz Biotechnology; sc-11415; 1:500; discontinued); Anti-VDAC1 (20B12AF2) mouse monoclonal antibody (Abcam; 14734; 1:500); Anti- $\beta$-actin (8H10D10) mouse monoclonal antibody (Cell Signaling Technology; 3700; 1:500). Secondary antibodies including anti-mouse IgG (NA931V) and anti-rabbit IgG (NA934V) were obtained from GE Healthcare. Detailed antibody validation profiles are available on the websites of the companies the antibodies were sourced from.

Tissue total lysate preparation - Samples were processed in the following lysis buffer: 10 mM HEPES-NaOH, pH 7.5, $150 \mathrm{mM} \mathrm{NaCl}, 1$ mM EGTA, 1\% Triton X-100, protease inhibitor cocktail, phosphatase inhibitor cocktail. After $20 \mathrm{~min}$ of incubation on ice, homogenates were spun at $14,000 \mathrm{rpm}$ for $20 \mathrm{~min}$ at $4{ }^{\circ} \mathrm{C}$. The supernatants correspond to the total lysates.

Randomization and blinding.-Animal/samples (mice) were assigned randomly to the various experimental groups, and mice were randomly selected for the analysis. In mouse imaging (Immunohistochemistry and data acquisition of electron microscopy), the performer(s) was (were) blinded to the experimental design. Additionally, a few biological replicates for mitochondrial transfer experiments, mitochondrial health assays and seahorse experiments were performed and analyzed by a person blinded to the experimental design.

Statistical analysis-Prism 8.0 (GraphPad Software) was used for the statistical analysis. Data shown are the mean \pm s.d. with $\mathrm{P}<0.05$ considered statistically significant. Group differences were analyzed with one-way analysis of variance (ANOVA) followed by Sidak's or Benjamini, Krieger, and Yekutieli correction multiple comparisons test or two-way ANOVA followed by Tukey's multiple comparisons test for multiple groups. No statistical methods were used to pre-determine sample sizes but our sample sizes are similar to those reported in previous publications ${ }^{9,18-19}$. Data distribution was assumed to be normal but this was not formally tested. No data/animals were excluded.

Data availability-The data that support the findings of this study are available from the corresponding author upon reasonable request.

\section{Supplementary Material}

Refer to Web version on PubMed Central for supplementary material.

\section{Acknowledgments}

In memory of Dr. Ben Barres, whose advise, and research inspired this study. This work was supported, in part, by Stanford Discovery Innovation Award and R01 HL52141 to D.M-R; Paul \& Daisy Soros Fellowship to P.S.M; a postdoctoral fellowship from the Australian National Health and Medical Research Council (GNT1052961) and the 
Glenn Foundation Glenn Award to S.A.L; RO1AG058047 to K.I.A and R35 HL135736 to G.W.D. The authors thank Narayan Raju and Steve Avolicino for assistance with immunohistochemistry and John Perrino for technical support with EM.

\section{References:}

1. Aguzzi A \& O’Connor T Protein aggregation diseases: pathogenicity and therapeutic perspectives. Nat Rev Drug Discov 9, 237-248 (2010). [PubMed: 20190788]

2. Hayakawa K, et al. Transfer of mitochondria from astrocytes to neurons after stroke. Nature 535, 551-555 (2016). [PubMed: 27466127]

3. Belanger M \& Magistretti PJ The role of astroglia in neuroprotection. Dialogues Clin Neurosci 11, 281-295 (2009). [PubMed: 19877496]

4. Chen Z \& Trapp BD Microglia and neuroprotection. J Neurochem 136 Suppl 1, 10-17 (2016). [PubMed: 25693054]

5. Crotti A \& Glass CK The choreography of neuroinflammation in Huntington's disease. Trends Immunol 36, 364-373 (2015). [PubMed: 26001312]

6. Heneka MT, et al. Neuroinflammation in Alzheimer's disease. Lancet Neurol 14, 388-405 (2015). [PubMed: 25792098]

7. Liu J \& Wang F Role of Neuroinflammation in Amyotrophic Lateral Sclerosis: Cellular Mechanisms and Therapeutic Implications. Front Immunol 8, 1005 (2017). [PubMed: 28871262]

8. Ransohoff RM How neuroinflammation contributes to neurodegeneration. Science 353, 777-783 (2016). [PubMed: 27540165]

9. Liddelow SA, et al. Neurotoxic reactive astrocytes are induced by activated microglia. Nature 541, 481-487 (2017). [PubMed: 28099414]

10. Itoh K, Nakamura K, Iijima M \& Sesaki H Mitochondrial dynamics in neurodegeneration. Trends Cell Biol. 23, 64-71 (2013). [PubMed: 23159640]

11. Reddy PH Increased mitochondrial fission and neuronal dysfunction in Huntington's disease: implications for molecular inhibitors of excessive mitochondrial fission. Drug Discov Today 19, 951-955 (2014). [PubMed: 24681059]

12. Joshi AU, et al. Inhibition of Drp1/Fis1 interaction slows progression of amyotrophic lateral sclerosis. EMBO Mol Med 10 (2018).

13. Khalil B \& Lievens JC Mitochondrial quality control in amyotrophic lateral sclerosis: towards a common pathway? Neural Regen Res 12, 1052-1061 (2017). [PubMed: 28852382]

14. Reddy PH, et al. Dynamin-related protein 1 and mitochondrial fragmentation in neurodegenerative diseases. Brain Res Rev 67, 103-118 (2011). [PubMed: 21145355]

15. Knott AB, Perkins G, Schwarzenbacher R \& Bossy-Wetzel E Mitochondrial fragmentation in neurodegeneration. Nat Rev Neurosci 9, 505-518 (2008). [PubMed: 18568013]

16. Qi X, Qvit N, Su YC \& Mochly-Rosen D A novel Drp1 inhibitor diminishes aberrant mitochondrial fission and neurotoxicity. J Cell Sci 126, 789-802 (2013). [PubMed: 23239023]

17. Grohm J, et al. Inhibition of Drp1 provides neuroprotection in vitro and in vivo. Cell Death Differ 19, 1446-1458 (2012). [PubMed: 22388349]

18. Joshi AU, Saw NL, Shamloo M \& Mochly-Rosen D Drp1/Fis1 interaction mediates mitochondrial dysfunction, bioenergetic failure and cognitive decline in Alzheimer's disease. Oncotarget 9, 6128-6143 (2018). [PubMed: 29464060]

19. Disatnik MH, et al. Potential biomarkers to follow the progression and treatment response of Huntington's disease. J Exp Med 213, 2655-2669 (2016). [PubMed: 27821553]

20. Guo X, et al. Inhibition of mitochondrial fragmentation diminishes Huntington's disease-associated neurodegeneration. J Clin Invest 123, 5371-5388 (2013). [PubMed: 24231356]

21. Mishra P \& Chan DC Metabolic regulation of mitochondrial dynamics. J Cell Biol 212, 379-387 (2016). [PubMed: 26858267]

22. Crotti A, et al. Mutant Huntingtin promotes autonomous microglia activation via myeloid lineagedetermining factors. Nat Neurosci 17, 513-521 (2014). [PubMed: 24584051] 
23. Sarkar S, et al. Mitochondrial impairment in microglia amplifies NLRP3 inflammasome proinflammatory signaling in cell culture and animal models of Parkinson's disease. NPJ Parkinsons Dis 3, 30 (2017). [PubMed: 29057315]

24. Liu Y, Hao W, Dawson A, Liu S \& Fassbender K Expression of amyotrophic lateral sclerosislinked SOD1 mutant increases the neurotoxic potential of microglia via TLR2. J Biol Chem 284, 3691-3699 (2009). [PubMed: 19091752]

25. Sondag CM, Dhawan G \& Combs CK Beta amyloid oligomers and fibrils stimulate differential activation of primary microglia. J Neuroinflammation 6, 1 (2009). [PubMed: 19123954]

26. Kornfeld OS, et al. Interaction of mitochondrial fission factor with dynamin related protein 1 governs physiological mitochondrial function in vivo. Sci Rep 8, 14034 (2018). [PubMed: 30232469]

27. Falchi AM, et al. Astrocytes shed large membrane vesicles that contain mitochondria, lipid droplets and ATP. Histochem Cell Biol 139, 221-231 (2013). [PubMed: 23108569]

28. Gogvadze V, Orrenius S \& Zhivotovsky B Multiple pathways of cytochrome c release from mitochondria in apoptosis. Biochim Biophys Acta 1757, 639-647 (2006). [PubMed: 16678785]

29. Ryan KJ, et al. A human microglia-like cellular model for assessing the effects of neurodegenerative disease gene variants. Sci Transl Med 9 (2017).

30. Cassidy-Stone A, et al. Chemical inhibition of the mitochondrial division dynamin reveals its role in Bax/Bak-dependent mitochondrial outer membrane permeabilization. Dev Cell 14, 193-204 (2008). [PubMed: 18267088]

31. Yates D Neurodegenerative disease: Factoring in astrocytes. Nat Rev Neurosci 16, 67 (2015). [PubMed: 25601774]

32. West AP, et al. Mitochondrial DNA stress primes the antiviral innate immune response. Nature 520, 553-557 (2015). [PubMed: 25642965]

33. Zhong Z, et al. New mitochondrial DNA synthesis enables NLRP3 inflammasome activation. Nature 560, 198-203 (2018). [PubMed: 30046112]

34. Chou SH, et al. Extracellular mitochondria in cerebrospinal fluid and neurological recovery after subarachnoid hemorrhage. Stroke 48, 2231-2237 (2017). [PubMed: 28663512]

35. Zussman B, Weiner G \& Ducruet A Mitochondrial transfer into the cerebrospinal fluid in the setting of subarachnoid hemorrhage. Neurosurgery 82, N11-N13 (2018). [PubMed: 29244132]

36. Hayakawa K, et al. Protective effects of endothelial progenitor cell-derived extracellular mitochondria in brain endothelium. Stem Cells 36, 1404-1410 (2018). [PubMed: 29781122]

37. Hayakawa K, et al. Extracellular mitochondria for therapy and diagnosis in acute central nervous system injury. JAMA Neurol 75, 119-122 (2018). [PubMed: 29159397]

38. Wilkins HM, et al. Extracellular mitochondria and mitochondrial components act as damageassociated molecular pattern molecules in the mouse brain. J Neuroimmune Pharmacol 11, 622 628 (2016). [PubMed: 27562848]

39. Labzin LI, Heneka MT \& Latz E Innate immunity and neurodegeneration. Annu Rev Med 69, 437-449 (2018). [PubMed: 29106805]

40. Glass CK, Saijo K, Winner B, Marchetto MC \& Gage FH Mechanisms underlying inflammation in neurodegeneration. Cell 140, 918-934 (2010). [PubMed: 20303880]

41. van Niel G, D'Angelo G \& Raposo G Shedding light on the cell biology of extracellular vesicles. Nat Rev Mol Cell Biol 19, 213-228 (2018). [PubMed: 29339798]

42. Lai RC, et al. MSC secretes at least $3 \mathrm{EV}$ types each with a unique permutation of membrane lipid, protein and RNA. J Extracell Vesicles 5, 29828 (2016). [PubMed: 26928672]

43. Taylor AR, Robinson MB, Gifondorwa DJ, Tytell M \& Milligan CE Regulation of heat shock protein 70 release in astrocytes: role of signaling kinases. Dev Neurobiol 67, 1815-1829 (2007). [PubMed: 17701989]

44. Hooper $\mathrm{C}$, et al. Wnt3a induces exosome secretion from primary cultured rat microglia. BMC Neurosci 13, 144 (2012). [PubMed: 23173708]

45. Glebov K, et al. Serotonin stimulates secretion of exosomes from microglia cells. Glia 63, 626-634 (2015). [PubMed: 25451814] 
46. Hessvik NP \& Llorente A Current knowledge on exosome biogenesis and release. Cell Mol Life Sci 75, 193-208 (2018). [PubMed: 28733901]

47. Islam MN, et al. Mitochondrial transfer from bone-marrow-derived stromal cells to pulmonary alveoli protects against acute lung injury. Nat Med 18, 759-765 (2012). [PubMed: 22504485]

48. Stevens JC, et al. Modification of superoxide dismutase 1 (SOD1) properties by a GFP tag-implications for research into amyotrophic lateral sclerosis (ALS). PLoS One 5, e9541 (2010). [PubMed: 20221404]

49. Minhas PS, et al. Macrophage de novo NAD(+) synthesis specifies immune function in aging and inflammation. Nat Immunol 20, 50-63 (2019). [PubMed: 30478397]

50. Valente AJ, Maddalena LA, Robb EL, Moradi F \& Stuart JA A simple ImageJ macro tool for analyzing mitochondrial network morphology in mammalian cell culture. Acta Histochem 119, 315-326 (2017). [PubMed: 28314612] 
a

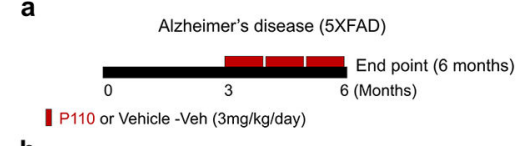

b

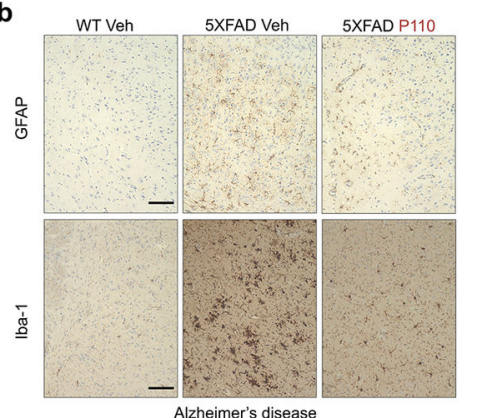

c

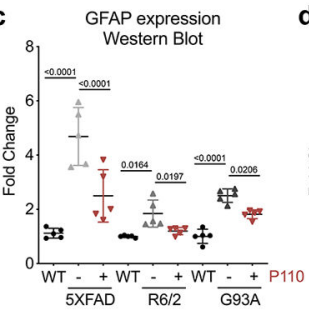

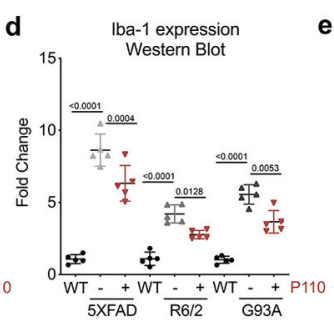

e
Huntington's disease (R6/2)
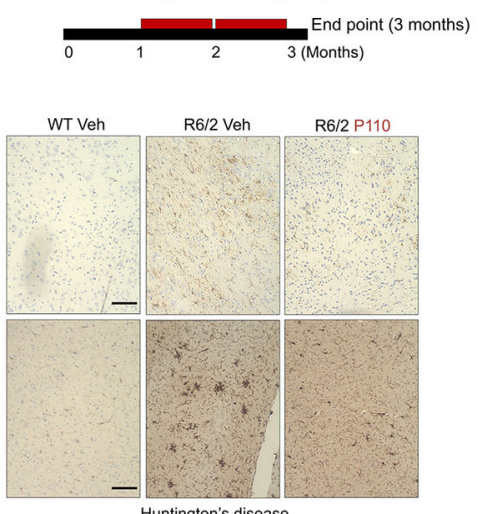

Amyotrophic lateral sclerosis (SOD1G93A)
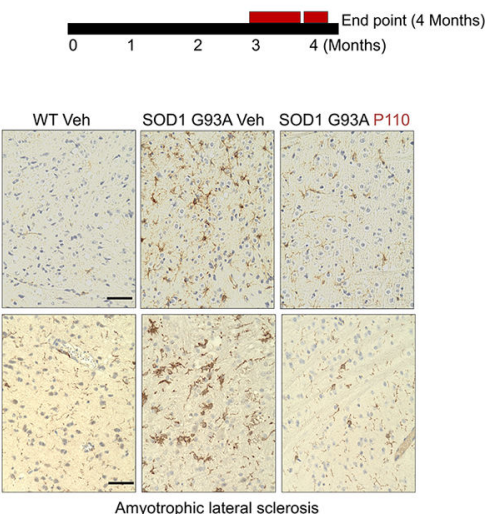

Amyotrophic lateral sclerosis
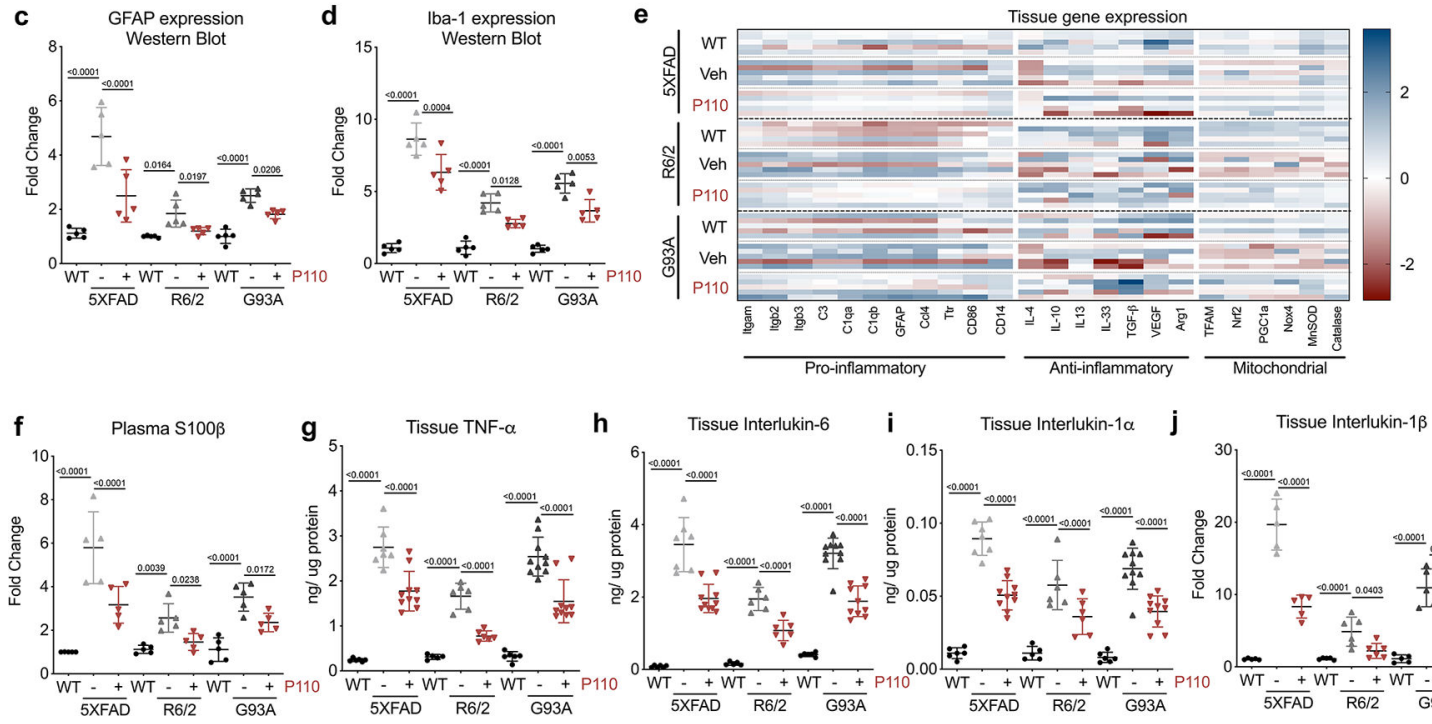

Tissue Interlukin-1 $\beta$

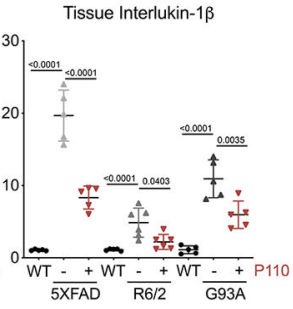

Figure 1. Inhibition of Drp1/Fis1-mediated mitochondrial fission in vivo reduces sustained microglia and astrocytes activation and subsequent pro-inflammatory response in three models of neurodegenerative diseases in mice

(a) Age of initiation and length of sustained treatment (in months) with P110 or vehicle (Veh; dark red rectangles in the scheme) in mouse models of Alzheimer's disease (AD; 5XFAD), Huntington's disease (HD; R6/2) and amyotrophic lateral sclerosis (ALS; SOD1G93A). (b) Representative sections of hippocampus, striatum and spinal cord immunostained for glial fibrillary acidic protein (GFAP) and ionized calcium-binding adapter molecule 1 (Iba-1) in 5xFAD AD mice (markers of astrocytes and microglia activation, respectively), in R6/2 HD mice and SOD1 G93A ALS mice, respectively, treated with vehicle or P110, at $3 \mathrm{mg} / \mathrm{kg} /$ day for the time indicated in the red bars in (a).

Representative of $n=5$ mice per group; 2 sections per mouse. Immunohistochemistry of brains of the corresponding wildtype (WT) mice are provided on the left of each mouse model. Scale bar: $50 \mu \mathrm{m}$. Note: The tissue samples were from the mice used in our previous publications $^{12,18,19}$. (c) GFAP and (d) Iba-1 protein levels (determined by quantitating immunostaining) in the brain of each mouse model, presented as ratio to the corresponding WT mice. (e) Expression profiles for select pro- and anti-inflammation-related genes and mitochondrial biogenesis-related genes as analyzed by qPCR in the respective tissue 
samples. Blue and red denote upregulated and downregulated genes, respectively ( $n=5$ per mice, of 2 technical repeats). (f) Plasma concentrations of $\mathrm{S} 100 \beta$ protein, a peripheral marker of astrocyte activation and blood brain barrier integrity ( $n=5$ per mice group). (g-j) Protein levels of tumor necrotic factor a (TNFa), interleukin-6 (IL-6), IL-1 $\alpha$ and IL-1 $\beta$ in tissue, in the respective tissue samples ( $n=5$ per mice group). Probability by one-way ANOVA and Benjamini, Krieger, and Yekutieli correction for multiple testing between each treatment group as above. All data are mean \pm s.d. 


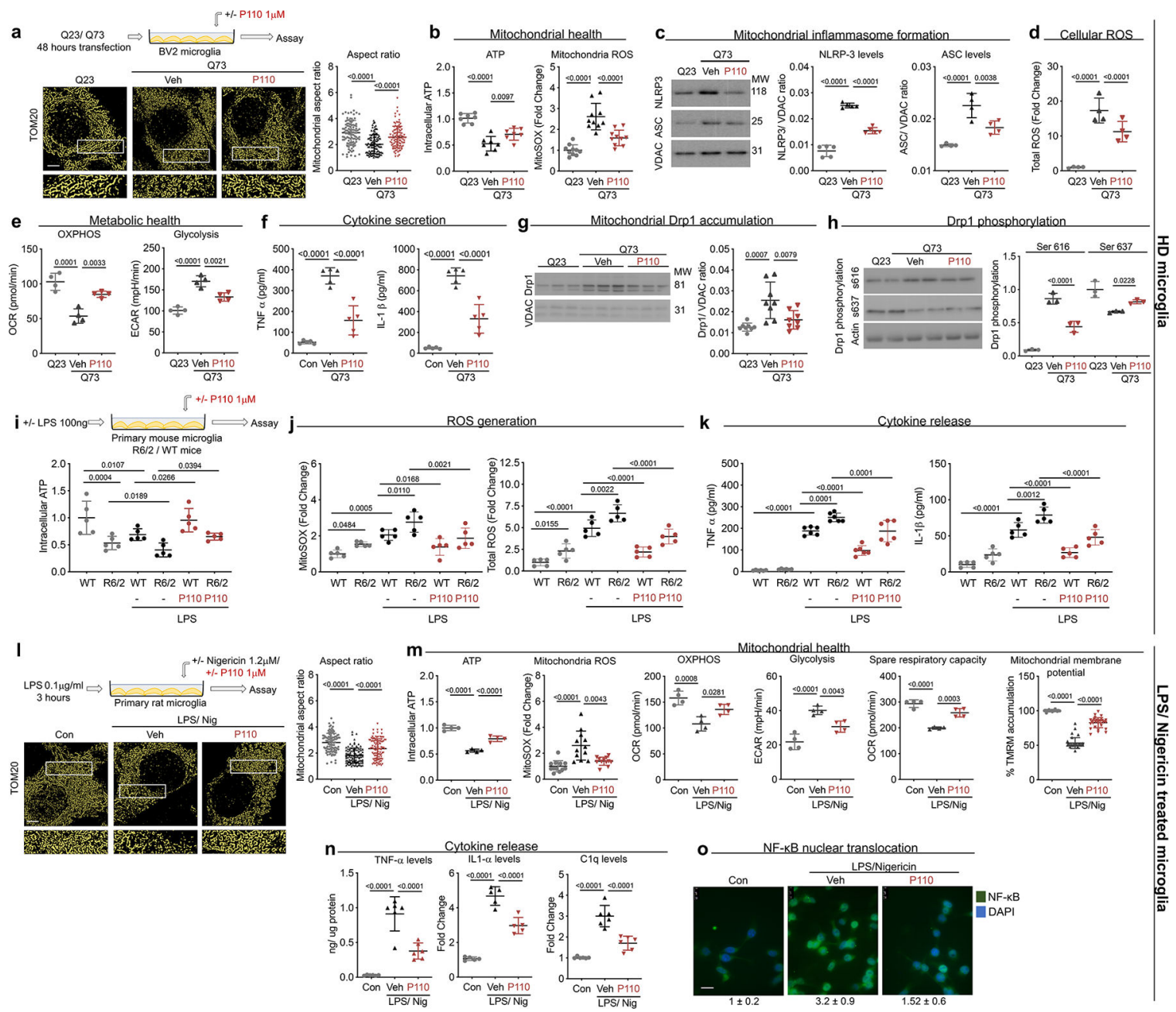

Figure 2. Drp1/Fis1-mediated mitochondrial fragmentation and dysfunction are required to induce microglial inflammatory response in a model of HD (induced by cytotoxic poly glutamine chain, Q73) or LPS activation, in culture.

(a) Protocol of treatment for panels a-h (top) and representative photomicrographs of rat BV2 microglia, transiently expressing 23 poly glutamine repeats (Q23; control) or Q73 (HD model) for 48 hours, then treated with vehicle or P110 ( $1 \mu \mathrm{M} /$ added once 24 hours after transfection in serum and antibiotic free DMEM and their mitochondria stained with antiTOM20 (pseudo-color in yellow - for microglia); after 24 hours. Mitochondrial aspect ratio was quantified using a macro in Fiji (ImageJ) ${ }^{50}$ (b) Intracellular ATP levels and mitochondrial ROS levels quantified after 16 hours. (c) Levels of inflammasome components, nucleotide-binding oligomerization domain, leucine rich repeat and pyrin domain-containing 3 (NLRP3) and apoptosis-associated speck-like protein containing a CARD (ASC), present in mitochondrial fractions of Q23 and Q73 expressing BV2 microglia after 24 hours. (d) Total cellular ROS levels were quantified after 24 hours. (e) Oxidative phosphorylation (OXPHOS) and glycolysis rate (using Seahorse) measured in BV2 mouse microglia cell line transiently expressing Q23 or Q73 for 48 hours (HD model) and treated without or with P110 ( $1 \mu \mathrm{M}$ every 24 hours) (f) TNFa and Il-1 $\beta$ levels in cell supernatants from Q23- or Q73-expressing BV2 microglia, treated as in (a) after 24 hours. (g) 
Mitochondria-associated Drp1 levels in the above BV2 cells, as a measure of Drp1 activation and (h) Drp1 phosphorylation levels at S616-Drp1 (a phosphorylation site correlating with Drp1 activation ${ }^{21}$ ) or -S637-Drp1 (a site correlating with Drp1 inhibition ${ }^{21}$ ) in Q23- or Q73-expressing BV2 microglia, treated as in (a) after 24 hours. (i) Above, the protocol of treatment for panels i-k and intracellular ATP levels in primary microglia from R6/2 or WT mice cultured and then treated with vehicle or P110 $(1 \mu \mathrm{M})$ and stimulated without or with LPS (10ng/ml) for 24 hours in serum and antibiotic free DMEM. (j) Mitochondrial ROS levels and total ROS levels quantified in R6/2 or WT primary mouse microglia treated as in (i). (k) Levels of released TNFa and IL- $1 \beta$ into the culture media of primary mouse microglia cells treated as in (i). (l) Protocol of treatment for panels 1-o (top) and photomicrographs of primary rat microglia primed with LPS $(10 \mathrm{ng} / / \mathrm{ml})$ for 3 hours followed by nigericin $(1.2 \mu \mathrm{M})$-treatment (LPS/Nig) in the presence or absence of P110 (1 $\mu \mathrm{M}$, added 30 minutes prior to LPS) treated for 21 hours in serum and antibiotic free DMEM and mitochondrial aspect ratio was quantified. (m) Markers of microglial mitochondrial health including intracellular ATP levels, mitochondrial ROS levels, OXPHOS, rate of glycolysis, spare respiratory capacity and mitochondrial membrane potential were determined in these control or LPS-primed nigericin-treated microglia. (n) Levels of TNFa and IL-1 $a$ in culture media, and C1q RNA levels in these controls or LPS-primed nigericintreated primary rat microglia in the absence or presence of P110. (o) Nuclear translocation of Nuclear factor kappa-light-chain-enhancer of activated $\mathrm{B}$ cells $(\mathrm{NF \kappa B})$ in primary rat microglia, treated as in (1). Values underneath are fold change of NFKB staining. Probability was calculated by one-way ANOVA (with Tukey's post hoc test). All graphs represent mean \pm s.d. Scale bar in a, 1 is $5 \mu \mathrm{m}$ and in o is $50 \mu \mathrm{m}$. All experiments were performed in biologically independent replicates. a: $n=3$, each point indicates single mitochondria; $b: n=7$

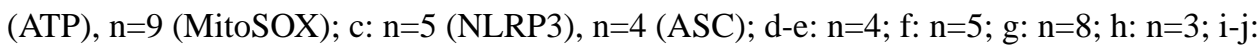
$\mathrm{n}=5 ; \mathrm{k}: \mathrm{n}=6$ (TNFa), $\mathrm{n}=5$ (IL-1 $\beta$ ); $1: \mathrm{n}=3$, each point indicates single mitochondria; $\mathrm{m}: \mathrm{n}=4$ (ATP), $n=12$ (MitoSOX), n=4 (OXHPHOS, Glycolysis, Spare Capacity), n=20 (TMRM); n: $n=6 ; o: n=3$. 


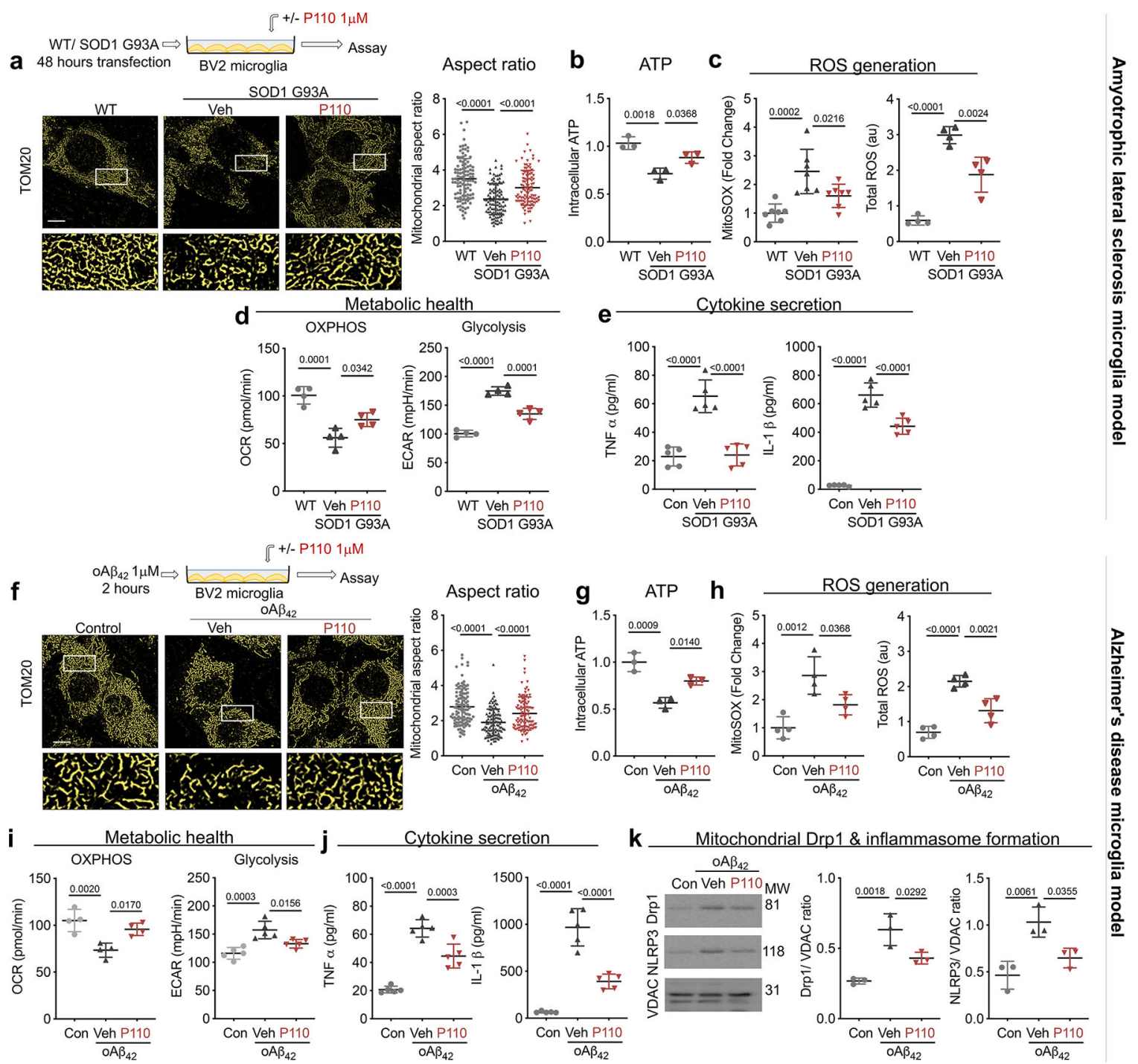

Figure 3. Drp1/Fis1-mediated mitochondrial fragmentation and dysfunction are required to induce microglial inflammatory response in a model of ALS (by expressing cytotoxic SOD G93A) or in a model of $\mathrm{AD}$ (induced by treatment with $\mathrm{oA} \beta_{1-42}$ ), in culture.

(a) Protocol of treatment for panels a-e (top) and representative photomicrographs (provided in pseudo-color in yellow, for microglia) and mitochondrial aspects in BV2 mouse microglia cell line transiently expressing human WT SOD1 (WT) or SOD1 G93A mutant (as an ALS model) for 48 hours treated as indicated. Intracellular ATP (b), ROS levels (c), and (d) mitochondrial oxygen consumption rate (OCR) and extracellular acidification rate (ECAR), measures of mitochondrial OXPHOS capacity and glycolysis, as determined using Seahorse, in BV2 expressing WT or mutant SOD1, as an ALS model after 24 hours in serum and antibiotic free DMEM. (e) Levels of released TNFa and IL-1 $\beta$ the culture media of the BV2 cells treated as in (a) were determined. (f) Protocol of treatment for panels f-k (top) and representative photomicrographs (pseudo-color in yellow, for microglia) and mitochondrial aspects of BV2 microglia treated with or without oligomeric $A \beta_{42}\left(\mathrm{oA} \beta_{42}\right)(1 \mu \mathrm{M})$ for 24 hours in the presence/absence of P110 ( $1 \mu \mathrm{M}$ added once together with oA $\left.\beta_{42}\right)$ in serum and antibiotic free DMEM as a model of AD. Intracellular ATP (g), ROS levels (h), and (i) 
metabolic health in BV2 microglia treated with or without $\left.\mathrm{oA} \beta_{42} ; 1 \mu \mathrm{M}\right)$ for $24 \mathrm{hrs}$ in defined medium in the presence or absence of $\mathrm{P} 110(1 \mu \mathrm{M}$, added 15 minutes prior to $\mathrm{oA} \beta_{42}$ ). (j) Levels of released TNFa and IL-1 $\beta$ the culture media of cells treated as in (f) were determined. (k) Mitochondrial Drp1 levels and NLRP3 levels in BV2 microglia, treated with or without oligomeric $\mathrm{oA} \beta_{42}(1 \mu \mathrm{M})$ for 24 hours in the presence/absence of P110 ( $1 \mu \mathrm{M}$ added once together with $\left.\mathrm{oA} \beta_{42}\right)$ in serum and antibiotic free DMEM, as a model of AD. Probability was calculated by one-way ANOVA (with Tukey's post hoc test). All graphs represent mean \pm s.d. Scale bar: $5 \mu \mathrm{m}$. All experiments were performed in biologically independent replicates. $\mathbf{a}: n=3$, each point indicates single mitochondria; $\mathbf{b}: n=3$ c: $n=7$ (MitoSOX), $n=4$ (Total ROS); d: $n=4 ; \mathbf{e : ~} n=5 ; \mathbf{f :} n=3$, each point indicates single mitochondria; $\mathbf{g}: \mathrm{n}=3 ; \mathbf{h}: \mathrm{n}=4 ; \mathbf{i}: \mathrm{n}=4$ (OXPHOS), $\mathrm{n}=5$ (Glycolysis); $\mathbf{j}: \mathrm{n}=5 ; \mathbf{k}: \mathrm{n}=3$. 

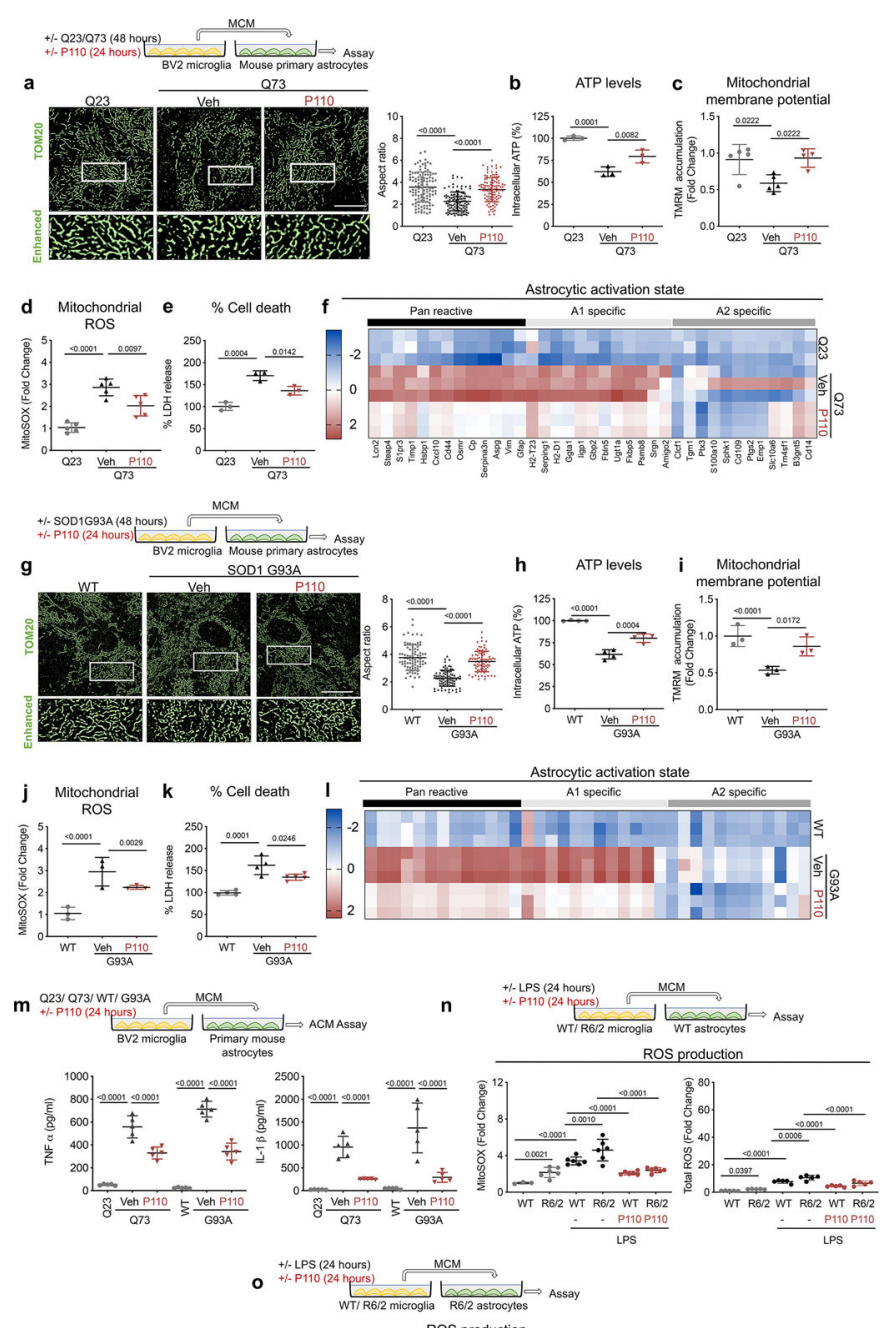

Figure 4. Conditioned media of activated microglia with mitochondrial dysfunction propagate astrocyte activation to A1 pro-inflammatory state and dysfunction of mitochondria in cultured astrocytes, using multiple models of neurodegenerative diseases.

(a) Protocol of the experiment in panels a-f (top) and representative photomicrographs of mouse primary astrocytes treated with conditioned media from activated BV2 microglia (referred to microglial conditioned media or MCM from here on) expressing a nonpathological poly-glutamine repeats, Q23 (control), or a pathological poly-glutamine repeats, Q73 (for 48 hrs; HD model) and treated with vehicle or P110 for $24 \mathrm{hrs}$. The MCM was added to astrocytes for 24 hours and astrocytes were then stained with anti-TOM20 (provided in pseudo-color green, for astrocytes) and mitochondrial aspect was determined as in (Fig. 1c). (b) Intracellular ATP levels in astrocytes were determined after 24 hours incubation with MCM, and (c) mitochondrial membrane potential and (d) mitochondrial ROS levels were determined at 6 hours after MCM treatments of the astrocytes. (e) LDH release from the astrocytes was measured 48 hours after MCM treatments. (f) Heat map of 
RNA transcripts in the astrocytes 24 hours after MCM treatments. (g) Protocol of the experiment in panels g-1 (top) and representative photomicrographs of mouse primary astrocytes (pseudo-color in green) treated for 12 hours with supernatant from BV2 microglia, expressing SOD1WT or SOD1G93A (MCM). The astrocytes were then stained with anti-TOM20 and mitochondrial aspect was determined as in (Fig. 1c). (h) Intracellular ATP levels at 24 hours, (i) mitochondrial membrane potential, using TMRM, and (j) mitochondrial ROS levels, using MitoSOX, were determined 6 hours after initiation of treatment with MCM. (k) LDH release measured 48 hours after MCM treatment. (l) Heat map of RNA transcripts of primary astrocytes primed by treatment with MCM. (m) Levels of released TNFa and IL- $1 \beta$ the culture media of cells treated as in (a and g) were determined. (n) Protocol of the experiment and mitochondrial ROS levels and total ROS levels were measured in primary astrocytes isolated from WT mice were treated with supernatant from WT/R6/2 mouse microglia, as in Fig. 2i. (o) Protocol of the experiment and mitochondrial ROS levels and total ROS levels were measured in primary astrocytes isolated from R6/2 mice were treated with supernatant from WT/R6/2 microglia treated as in Fig. 2i. Data were evaluated by one-way ANOVA and Holm-Sidak's multiple comparisons test for multiple testing between each treatment group. All graphs represent mean \pm s.d. Scale bar: $20 \mu \mathrm{m}$. Note that P110 treatment in the studies shown here and in Figs. 4-6 was restricted to the microglia. As the half-life of the peptide is $>30$ minutes, no peptide remained in the microglial conditioned media to directly affect the astrocytes or the neurons. All experiments were performed in biologically independent replicates. a: $n=3$, each point indicates single mitochondria; b: $\mathrm{n}=3$; $\mathbf{c - d}: \mathrm{n}=5 ; \mathbf{e}: \mathrm{n}=3 ; \mathbf{f}: \mathrm{n}=2 ; \mathbf{g}: \mathrm{n}=3$, each point indicates single mitochondria; $\mathbf{h}: \mathrm{n}=4 ; \mathbf{i}-\mathbf{j}: \mathrm{n}=4 ; \mathbf{k}: \mathrm{n}=4 ; \mathbf{l}: \mathbf{n}=2 ; \mathbf{m}: \mathrm{n}=5 ; \mathbf{n}: \mathrm{n}=6 ; \mathbf{o}: \mathrm{n}=5$. 

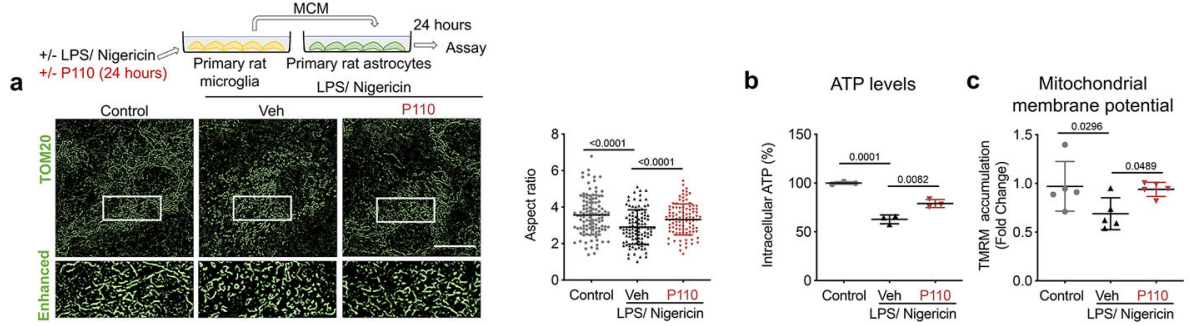

d Mitochondrial

e $\%$ Cell death
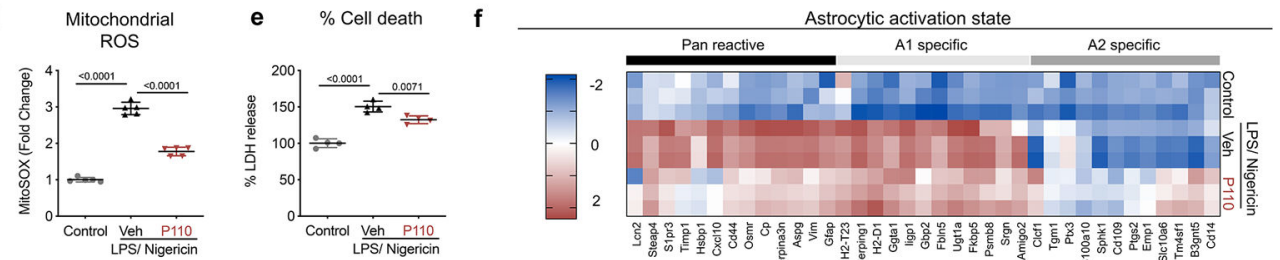

$+/-\mathrm{TNF}-\alpha / \mathrm{IL}-1 \alpha / \mathrm{C} 1 \mathrm{~d}$ $\stackrel{24 \text { hours }}{\rightleftarrows \text { Assay }}$

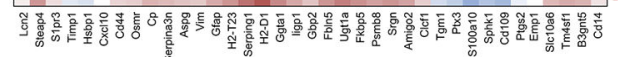

g
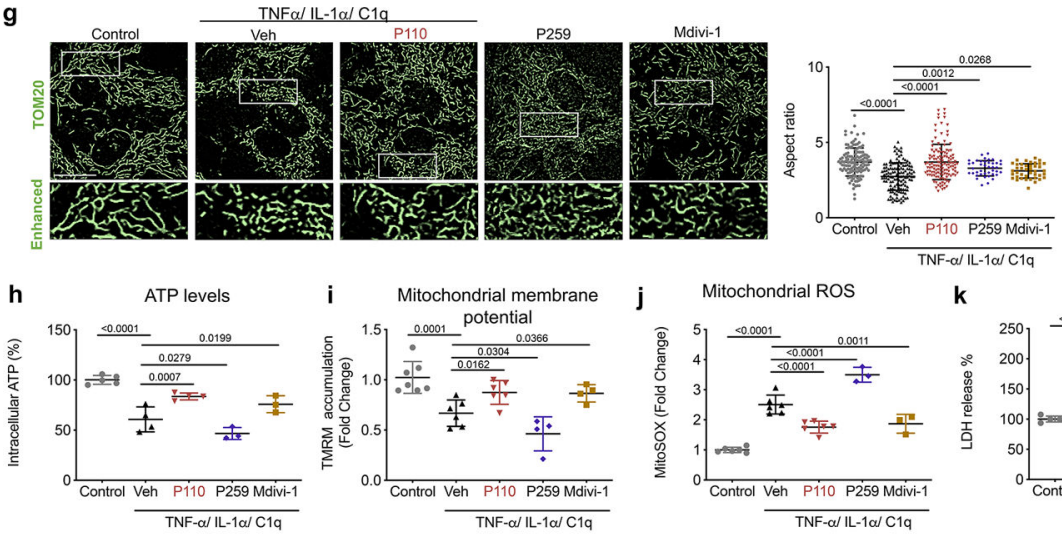

k $\quad \%$ Cell death

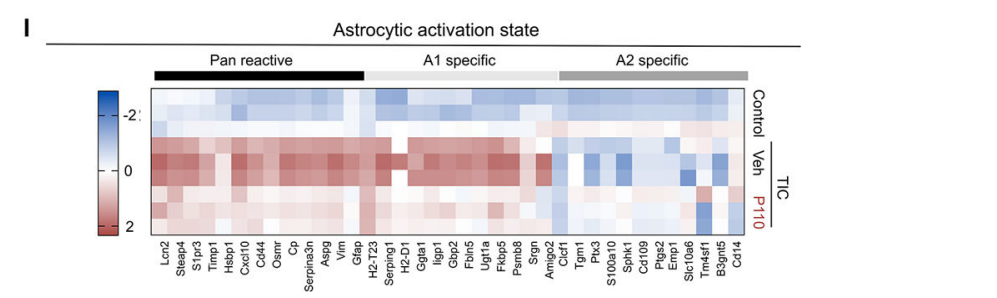

Figure 5: Mitochondrial excessive fission and dysfunction in activated astrocytes

(a) Schematic of the experimental design using primary mouse astrocytes treated with conditioned media of LPS/Nigericin-activated mouse primary microglia (MCM) (top). Representative photomicrographs of primary rat astrocytes (pseudo-color in green) treated with MCM for 24 hours and then stained with anti-TOM20 and mitochondrial aspect was determined. (b) Intracellular ATP determined at 24 hours, and (c) mitochondrial membrane potential and (d) mitochondrial ROS levels determined 12 hours after MCM treatment. (e) LDH release measured 48 hours after MCM treatment. (f) Heat map of RNA transcripts in these astrocytes. (g) Schematic of the experimental design using primary rat astrocytes treated directly with combination of TNFa, Il-1a, and C1q without or with P110 or P259 (1 $\mu \mathrm{M}$, added once, 15 minutes prior) or $20 \mu \mathrm{M}$ Mdivi-1 (added once, 15 minutes prior) and then incubate for 24 hours in serum and antibiotic free DMEM (top). Representative photomicrographs of primary mouse astrocytes (pseudo-color in green) as in the scheme and 
then stained with anti-TOM20 and mitochondrial aspect was determined. (h) Intracellular ATP at 24 hours, (i) Mitochondrial membrane potential at 24 hours, (j) mitochondrial ROS at 24 hours, (k) LDH release measured at 48 hours and (l) heat map of RNA transcripts of treated rat primary astrocytes at 24 hours were determined as above. Data were evaluated by one-way ANOVA and Holm-Sidak's multiple comparisons test for multiple testing between each treatment group. All graphs represent mean \pm s.d. Scale bar: $20 \mu \mathrm{m}$. All experiments were performed in biologically independent replicates. $\mathbf{a}: \mathrm{n}=3$, each point indicates single mitochondria; b: $\mathrm{n}=3 ; \mathbf{c}-\mathbf{d}: \mathrm{n}=5 ; \mathbf{e}: \mathrm{n}=4 ; \mathbf{f}: \mathrm{n}=2 ; \mathbf{g}: \mathrm{n}=3$, each point indicates single mitochondria; h: $\mathrm{n}=5$ (Control, Veh, P110) n=3 (P259, Mdivi-1); i: $\mathrm{n}=7$ (Control, Veh, P110) n=4 (P259, Mdivi-1); j: n=6 (Control, Veh, P110) n=3 (P259, Mdivi-1); k: n=5 (Control, Veh, P110) n=3 (P259, Mdivi-1); l: $n=2$. 

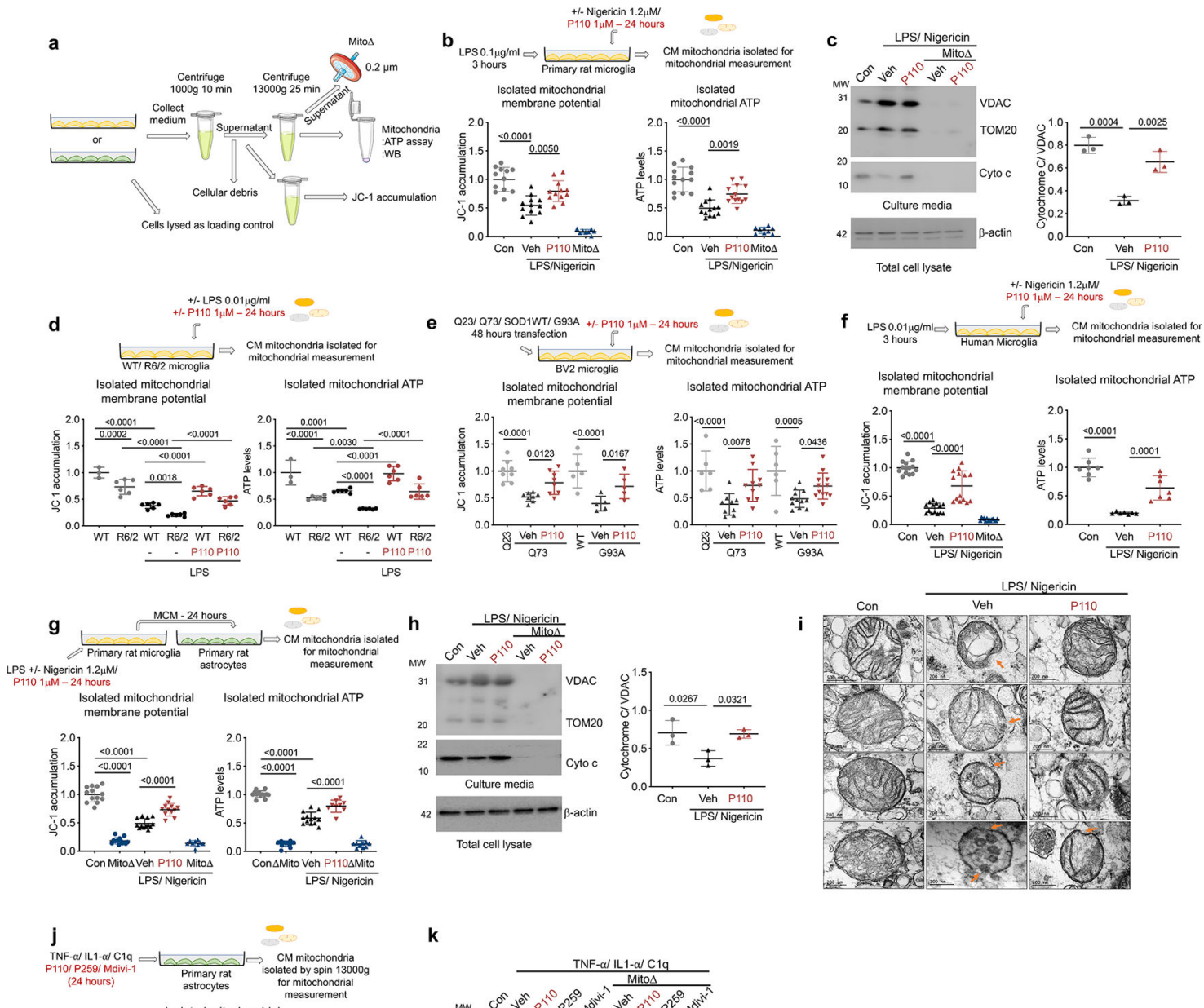

k Isolated mitochondrial
membrane potential
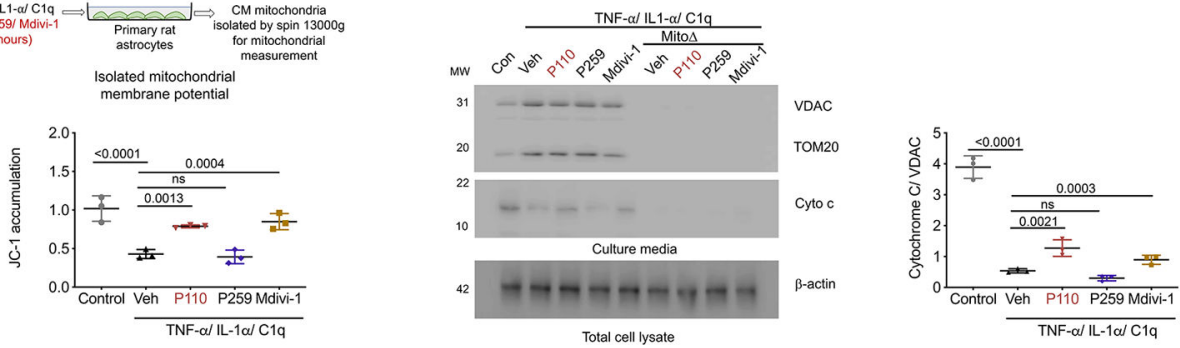

Figure 6. Dysfunctional extracellular mitochondria released from activated primary rat and mouse microglia or astrocytes or human primary monocyte-derived microglial cells in a Drp1/ Fis1-specific manner.

(a) Protocol of the experiment in panels a-c (top). (b) Levels of mitochondrial membrane potential and ATP in mitochondria-enriched pellet collected from cultured conditioned media of rat primary microglia, as depicted in the scheme. Extracellular mitochondria were removed from the media, using $0.2 \mu \mathrm{m}$ filters; Mito $\Delta$. (c) Integrity of the extracellular mitochondria was analyzed for markers of outer-membrane proteins (TOM20 and VDAC) and cytochrome $\mathrm{c}$, an inter-membrane space protein, that readily leaks out when outer mitochondrial membrane integrity is compromised. $\beta$-actin of the cell extracts from the corresponding plates was used as a loading control. (d) Protocol of the experiment and levels of mitochondrial membrane potential and ATP in mitochondria-enriched pellet collected from cultured conditioned media of primary microglia isolated from WT/R6/2 mice treated without or with P110 (1 $\mu \mathrm{M}$, added once 15 minutes prior to LPS $0.5 \mu \mathrm{g} / \mathrm{ml})$ and for 24 
hours in serum and antibiotic free DMEM. (e) Protocol of the experiment and levels of mitochondrial membrane potential and ATP in mitochondria-enriched pellet collected from cultured conditioned media of transiently expressing Q23/Q73 (control and a HD model, respectively) or human WT SOD1 or SOD1 G93A mutant (control and an ALS model, respectively) for 48 hours treated without or with P110 (1 $\mu \mathrm{M})$. (f) Protocol of the experiment for panels, levels of mitochondrial membrane potential and ATP in mitochondrial pellet collected from cultured conditioned media of model of human microglia. Extracellular mitochondria were removed from the media, using $0.2-\mu \mathrm{m}$ filters; Mito $\Delta$, as in (a). (g) Membrane potential and ATP levels of the extracellular mitochondria released from rat primary astrocytes treated with rat microglial conditioned media.

Extracellular mitochondria were removed from the media, using 0.2- $\mu$ m filters; Mito $\Delta$. (h) The integrity of the extracellular astrocytic mitochondria was analyzed as in (c). Extracellular mitochondria were removed from the media, using 0.2- $\mu \mathrm{m}$ filters; Mito $\Delta$. (i) The morphology and structural organization of the extracellular mitochondria were examined in electron microscope micrographs of pelleted mitochondria from the conditioned media of astrocytes. Scale bar: 200-500 nm, as indicated. (j) Protocol and membrane potential of the extracellular mitochondria released from primary rat astrocytes treated with as in Fig 5a; in addition to the Drp1/Fis1-selective inhibitor, cells were also treated with P259, a Mff/Drp1-selective inhibitor ${ }^{26}(1 \mu \mathrm{M})$ or with Mdivi-1 $(20 \mu \mathrm{M})$, a Drp1 GTPase inhibitor ${ }^{30}$. (k) The integrity of the extracellular mitochondria was analyzed as in (c) and represented as a ratio to $\beta$-actin in total lysate. Extracellular mitochondria were removed from the media, using $0.2-\mu \mathrm{m}$ filters; Mito $\Delta$, as in (a). Data were evaluated by oneway ANOVA and Holm-Sidak's multiple comparisons test for multiple testing between each treatment group. All graphs represent mean \pm s.d. All experiments were performed in biologically independent replicates. b: $n=12 ; \mathbf{c}: n=3 ; \mathbf{d}: n=9 ; \mathbf{e}: n=7 ; \mathbf{f}: n=13$ (JC-1), $n=7$ (ATP); $\mathbf{g}: \mathrm{n}=13$ (JC-1), $\mathrm{n}=9$ (ATP); h: $\mathrm{n}=3 ; \mathbf{i}: \mathbf{n}=2 ; \mathbf{j}-\mathbf{k}: \mathbf{n}=3$. 


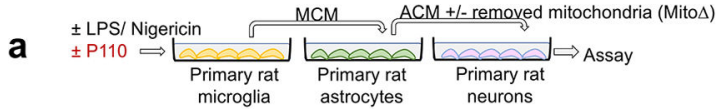

b
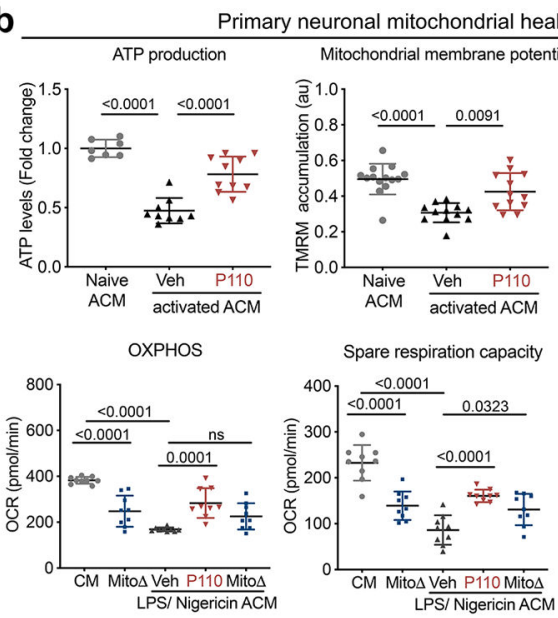

Spare respiration capacity
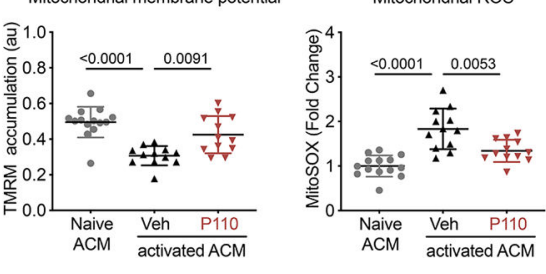

Maximal respiration
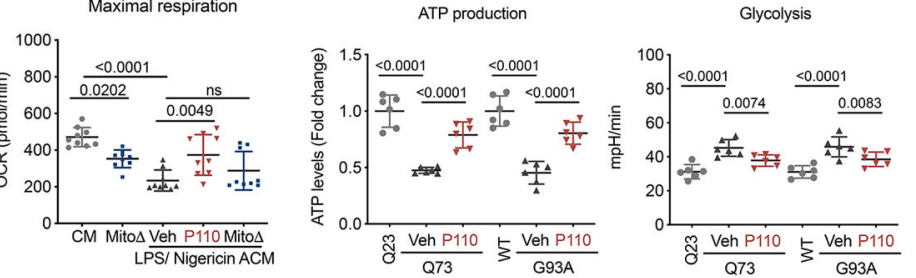

d
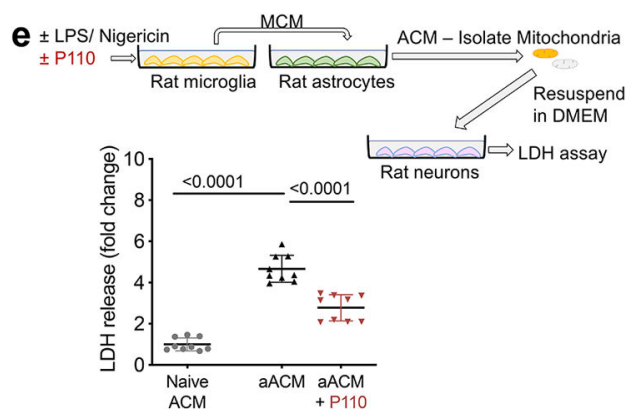

Figure 7. Propagation of mitochondrial dysfunction and cell death from activated microglia to neurons through astrocytes, by the released (extracellular) glial mitochondria.

(a) Schematic of the experimental design. (b) ATP levels, mitochondrial membrane potential using TMRM, mitochondrial ROS using MitoSOX, OCR measurement for OXPHOS, spare respiratory capacity and maximal respiration using seahorse, in rat primary cortical neurons treated with activated astrocyte conditioned medium (aACM; as in Supplementary Fig. 3a) that were activated by media from microglia treated as indicated by LPS/nigericin in the presence or absence of P110 $(1 \mu \mathrm{M})$. Extracellular mitochondria from the microglial conditioned media were removed prior to transferring the microglial-conditioned media to the astrocytes, using 0.2- $\mu$ m filters; Mito $\Delta$. OCR, TMRM and MitoSOX were measured at 24 hrs. (c) Oxygen consumption of mouse primary neurons 24 hour after exposure to conditioned medium of astrocytes (ACM) that were treated with conditioned media of BV2 microglia, expressing Q73, or SOD1 G93A, or the corresponding controls; in the absence or presence of P110. (d) Scheme of the experimental protocol (top) and LDH release (a marker of neuronal cell death) from primary cortical rat neurons treated with rat activated ACM (aACM) and the consequence of removal of extracellular mitochondria (filtration through $0.2-\mu \mathrm{m}$ filters; Mito $\Delta$ ) released from the astrocytes, or removal of extracellular DNA released from the aACM (using DNase). (e) Scheme of the experimental protocol (top) and rat neuronal cell death (LDH release) induced by transferring of enriched preparation of extracellular mitochondria isolated from primary rat aACM that were treated by conditioned 
media (MCM) of primary rat microglia treated with LPS/Nig together with or without P110. Data were evaluated by one-way ANOVA and Holm-Sidak's multiple comparisons test for multiple testing between each treatment group. All graphs represent mean \pm s.d. All experiments were performed in biologically independent replicates. b: $n=7$ (naïve), $n=10$ (activated ACM) (ATP), $\mathrm{n}=14$ (naïve), $\mathrm{n}=12$ (activated ACM) (TMRM, MitoSOX), $\mathrm{n}=9$ (Seahorse); c: $n=6 ; \mathbf{d}: \mathrm{n}=13 ; \mathbf{e}: \mathrm{n}=9$. 

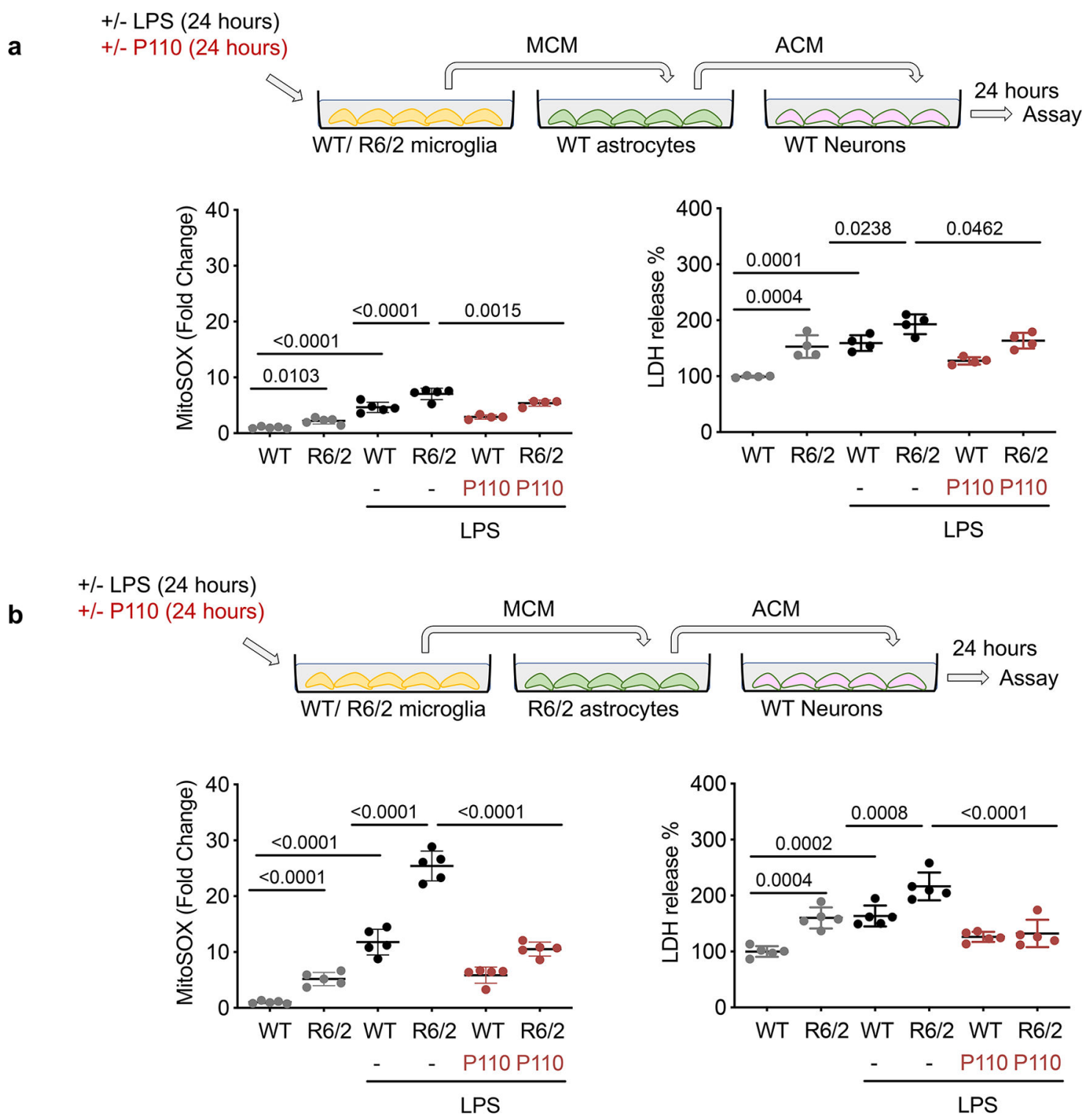

Figure 8. Drp1/Fis1-dependent propagation of neuronal mitochondrial dysfunction and neuronal cell death from R6/2 microglia to naïve mouse cortical neurons through WT or R6/2 astrocytes.

(a) Schematic of the experimental design using primary microglia (treated without or with LPS) that were isolated from R6/2 mice or their littermate WT mice. Mitochondrial ROS and LDH release in mouse primary cortical neurons treated with activated astrocyte conditioned medium (aACM) from WT mouse astrocytes. (b) The treatment protocol is as in (a), except that, in addition to the microglia, the primary astrocytes used were isolated from R6/2 mice, or the WT littermates, as indicated. All graphs represent mean \pm s.d. Data were evaluated by one-way ANOVA and Holm-Sidak's multiple comparisons test. All experiments were performed in biologically independent replicates. a: $n=5$ (MitoSOX), $n=4$ $(\mathrm{LDH}) ; \mathbf{b}: \mathrm{n}=5$. 